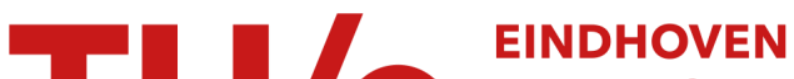 UNIVERSITY OF TECHNOLOGY
}

\section{Partial synchronization in diffusively time-delay coupled oscillator networks}

Citation for published version (APA):

Steur, E., Oguchi, T., Leeuwen, van, C., \& Nijmeijer, H. (2012). Partial synchronization in diffusively time-delay coupled oscillator networks. Chaos, 22, 043144-1/17. [043144]. https://doi.org/10.1063/1.4771665

DOI:

10.1063/1.4771665

Document status and date:

Published: 01/01/2012

Document Version:

Publisher's PDF, also known as Version of Record (includes final page, issue and volume numbers)

Please check the document version of this publication:

- A submitted manuscript is the version of the article upon submission and before peer-review. There can be important differences between the submitted version and the official published version of record. People interested in the research are advised to contact the author for the final version of the publication, or visit the $\mathrm{DOI}$ to the publisher's website.

- The final author version and the galley proof are versions of the publication after peer review.

- The final published version features the final layout of the paper including the volume, issue and page numbers.

Link to publication

\section{General rights}

Copyright and moral rights for the publications made accessible in the public portal are retained by the authors and/or other copyright owners and it is a condition of accessing publications that users recognise and abide by the legal requirements associated with these rights.

- Users may download and print one copy of any publication from the public portal for the purpose of private study or research.

- You may not further distribute the material or use it for any profit-making activity or commercial gain

- You may freely distribute the URL identifying the publication in the public portal.

If the publication is distributed under the terms of Article $25 \mathrm{fa}$ of the Dutch Copyright Act, indicated by the "Taverne" license above, please follow below link for the End User Agreement:

www.tue.nl/taverne

Take down policy

If you believe that this document breaches copyright please contact us at:

openaccess@tue.nl

providing details and we will investigate your claim. 


\section{Partial synchronization in diffusively time-delay coupled oscillator networks}

Erik Steur, Toshiki Oguchi, Cees van Leeuwen, and Henk Nijmeijer

Citation: Chaos 22, 043144 (2012); doi: 10.1063/1.4771665

View online: http://dx.doi.org/10.1063/1.4771665

View Table of Contents: http://chaos.aip.org/resource/1/CHAOEH/v22/i4

Published by the American Institute of Physics.

\section{Related Articles}

Synchronous rotation of the set of double pendula: Experimental observations Chaos 22, 047503 (2012)

Kuramoto model with coupling through an external medium

Chaos 22, 043139 (2012)

Horseshoes of periodically kicked van der Pol oscillators

Chaos 22, 043140 (2012)

Metastability and chimera states in modular delay and pulse-coupled oscillator networks

Chaos 22, 043131 (2012)

Outer synchronization between two complex dynamical networks with discontinuous coupling

Chaos 22, 043125 (2012)

\section{Additional information on Chaos}

\section{Journal Homepage: http://chaos.aip.org/}

Journal Information: http://chaos.aip.org/about/about_the_journal

Top downloads: http://chaos.aip.org/features/most_downloaded

Information for Authors: http://chaos.aip.org/authors

\section{ADVERTISEMENT}

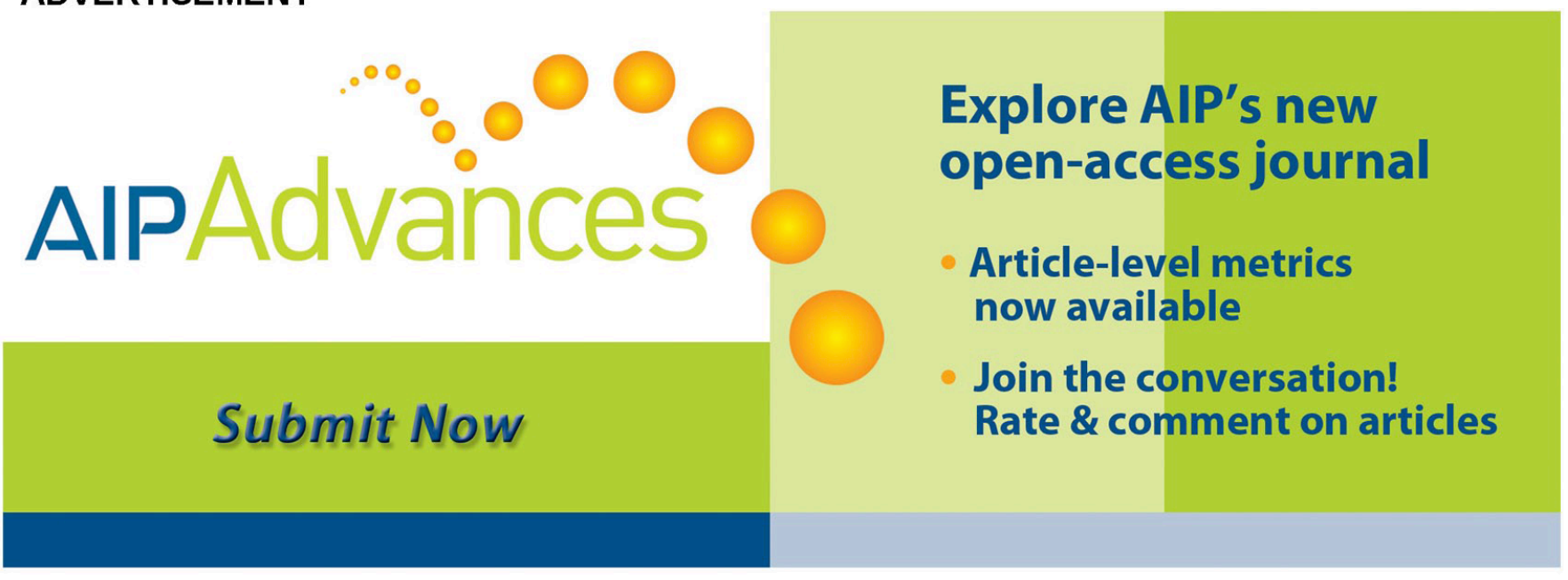




\title{
Partial synchronization in diffusively time-delay coupled oscillator networks
}

\author{
Erik Steur, ${ }^{1, a)}$ Toshiki Oguchi, ${ }^{2, b)}$ Cees van Leeuwen, ${ }^{3, c)}$ and Henk Nijmeijer ${ }^{1, d)}$ \\ ${ }^{1}$ Department of Mechanical Engineering, Eindhoven University of Technology, P.O. Box 513, \\ $5600 \mathrm{MB}$, Eindhoven, the Netherlands \\ ${ }^{2}$ Department of Mechanical Engineering, Graduate School of Science and Engineering, Tokyo Metropolitan \\ University, 1-1, Minami-Osawa, Hachioji-shi, Tokyo 192-0397, Japan \\ ${ }^{3}$ Faculty of Psychology and Educational Sciences, Research Group Experimental Psychology, Laboratory for \\ Perceptual Dynamics, KU Leuven, Tiensestraat 102, B-3000 Leuven, Belgium
}

(Received 17 September 2012; accepted 28 November 2012; published online 26 December 2012)

\begin{abstract}
We study networks of diffusively time-delay coupled oscillatory units and we show that networks with certain symmetries can exhibit a form of incomplete synchronization called partial synchronization. We present conditions for the existence and stability of partial synchronization modes in networks of oscillatory units that satisfy a semipassivity property and have convergent internal dynamics. (C) 2012 American Institute of Physics. [http://dx.doi.org/10.1063/1.4771665]
\end{abstract}

In the study of synchronization in oscillator networks where coupling is diffusive and allows for time-delays, the focus is on deriving conditions that guarantee synchronization of all units in the network. We considered the question what happens if full synchronization cannot be achieved. Will there be no collective behavior at all or might it be possible that partial synchronization occurs, i.e., that some, but not all, units synchronize? We show that if a network contains certain symmetries, then these symmetries identify modes of partial synchronization. We present conditions for the existence and stability of partial synchronization modes in networks of diffusive time-delay coupled oscillatory units. The results are supported by numerical simulations in several networks of diffusively time-delay coupled neural Hindmarsh-Rose oscillators.

\section{INTRODUCTION}

The study of synchronization in oscillator networks has received considerable attention over the last decades. One reason for this is the existence of many applications in physics, neuroscience, and biology. Examples include the simultaneous flashing of thousands of fireflies that gather in trees along the tidal rivers in Malaysia ${ }^{1,2}$ (see, Ref. 3 for a nice color picture), clusters of synchronized pacemaker neurons, which regulate our heartbeat, ${ }^{4}$ synchronized neurons in the olfactory bulb that allow to detect and distinguish between odors, ${ }^{5}$ and our circadian rhythm, which is synchronized to (more precisely, entrained to) the 24 -h day-night cycle. ${ }^{6,7}$ Synchronization has also found applications in diverse fields of engineering, such as platooning of vehicles, ${ }^{8}$ cooperation of robotic systems, ${ }^{9,10}$ and secure communication. ${ }^{11-13}$

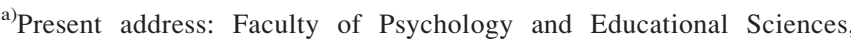
Research Group Experimental Psychology, Laboratory for Perceptual Dynamics, KU Leuven, Tiensestraat 102, B-3000 Leuven, Belgium. Electronic mail: erik.steur@ppw.kuleuven.be.

b)t.oguchi@tmu.ac.jp.

c)cees.vanleeuwen@ppw.kuleuven.be.

d)h.nijmeijer@tue.nl.
}

In this study, we focus on networks where the coupling is diffusive. Diffusive coupling is a linear coupling that is proportional to the difference of the output signals of the interacting units, cf. Refs. 14 and 15. This type of coupling arises in various areas of science arranging from physiology ${ }^{16,17}$ and neuroscience ${ }^{18-22}$ to electrical systems ${ }^{23,24}$ and mechanical engineering. ${ }^{25-28}$ In addition, we allow the coupling to contain time-delays, which can arise due to finite propagation speed of information and/or the time it takes to make decisions, cf. Ref. 29.

Synchronization in networks of diffusively time-delay coupled units, where synchronization is understood as the asymptotic matching of the states of all units in the network, has been investigated in, for instance, Refs. 27 and 30-39. The general conclusion is that in order to achieve synchronization, the coupling strength has at least to exceed a certain threshold value. On the other hand, there is evidence that full synchronization will generally not occur when time-delays are large. ${ }^{40,41}$ An important question is what happens if synchronization of all units in the network cannot be achieved, e.g., when the coupling is not strong enough and/or the time-delays are too large. One possible outcome, at least in principle, is that there is no coherent behavior at all. However, there is evidence that although full synchronization is not achieved, it is possible that some units of the network synchronize. For instance, in Ref. 42, it is shown that time-delay can induce the emergence of synchronized clusters in networks. In this paper, we will show that certain networks allow partial synchronization, the phenomenon where some but not all units in the network synchronize.

Partial synchronization in networks of non-delayed diffusively coupled oscillatory units has been investigated in, for instance, Refs. 43-46. This study extends the ideas presented in Refs. 44 and 45 to the case of general time-delayed diffusive coupling. The systematic approach of Refs. 44 and 45 identifies linear invariant manifolds defined by local and global symmetries in network the coupled dynamical units. (A linear manifold is called a linear invariant manifold if it is invariant with regards to the flow of the dynamical system.) Local symmetries are those symmetries that can be present in the units itself, cf. Refs. 44, 47, and 48. For example, the Lorenz system ${ }^{49}$ is a system with a local symmetry. ${ }^{44}$ Indeed, the Lorenz equations 


$$
\begin{aligned}
& \dot{x}_{1}=\sigma\left(x_{2}-x_{1}\right), \\
& \dot{x}_{2}=r x_{1}-x_{2}-x_{1} x_{3}, \\
& \dot{x}_{3}=-b x_{3}+x_{1} x_{2},
\end{aligned}
$$

are invariant under the change of coordinates $x \mapsto y$ with $y_{1}=-x_{1}, y_{2}=-x_{2}$ and $y_{3}=x_{3}$. Global symmetries are symmetries that are present in the network, hence it is possible to have global symmetries in networks of systems of any type. Because our main interest is in how partial synchronization emerges as function of coupling strength and/or timedelay, which are parameters of the network, we consider only global symmetries. We would like to add that the results presented in this paper can easily be extended to include local symmetries too. ${ }^{50}$ It is important to realize that local symmetries might influence the (synchronous) behavior of a network. See, Ref. 44 for an example.

In case of a global symmetry, i.e., if the network contains a certain symmetry, the symmetry must be present in the adjacency matrix $A$ of the network (and thus also in the Laplacian matrix $L$ ). Then, a rearrangement of (some of) the entries of $A$ will leave the network unchanged. Mathematically, the rearrangement of the entries of $A$ is described by the (pre)multiplication of $A$ with a permutation matrix. Recall that a permutation matrix is a matrix with exactly one entry equal to one in each row and each column and zeros everywhere else. Permutation matrices are orthogonal and form a group (with identity element $I$ ) under multiplication. It is shown in Ref. 44 that if there is a permutation matrix $\Pi$ that commutes with the Laplacian matrix $L$, i.e., $\Pi L=L \Pi$, then the set $\operatorname{ker}\left(I_{k n}-\Pi \otimes I_{n}\right)$ defines a linear invariant manifold for the network. Sufficient conditions were presented for this manifold to be asymptotically stable, which, in absence of full synchronization, obviously implies partial synchronization. In Ref. 45 , the assumption that $\Pi$ and $L$ commute is relaxed; it is proven that the result of Ref. 44 remains true if $\Pi$ and $L$ not commute but there exists a solution $X$ to the matrix equation $(I-\Pi) L=X(I-\Pi)$. (Obviously, if $\Pi$ and $L$ commute, $L=X$.) We show that the ideas presented in Refs. 44 and 45 are also useful to investigate partial synchronization in networks where the units interact via general time-delay diffusive coupling. By general diffusive coupling, we mean that we do not assume the coupling to be symmetric. (Symmetry of the coupling means that if unit $i$ couples to unit $j$ with a certain strength $a_{i j}$, then unit $j$ couples to unit $i$ with strength $a_{j i}=a_{i j}$.) To the best of our knowledge, there are only a few theoretical results on partial synchronization in network of time-delay diffusively coupled oscillatory units available in literature, see Refs. 42 and 51. The results presented in Refs. 42 and 51 are based on the master stability function, ${ }^{52}$ which is essentially a local analysis. The results that are presented in this paper hold, on the contrary, globally. In addition, our approach does not require a priori knowledge of the partially synchronized solutions, i.e., the solutions of the systems on a partial synchronization manifold, whereas knowing the partially synchronized solutions is essential for successful application of techniques based on the master stability function. Determining the partially synchronized solutions is, however, far from obvious since the dynamics on the partial synchronization manifold will not be autonomous (since the coupling functions do not vanish on the partial synchronization manifold). Of course, there is also a price to pay; our approach provides a qualitative description but no quantitative description of the partially synchronized dynamics, hence our approach does maybe not offer the best estimates of threshold values of coupling strength and/or time-delay that will give (partial) synchronization.

The remaining part of this paper is organized as follows. In Sec. II, we introduce the notation that will be used throughout the paper. In addition, we introduce the concepts of semipassivity and convergent systems, which will be used to guarantee boundedness of solutions of the whole network and convergence to the partially synchronized state. In Sec. III, we present our problem setting. Section IV presents conditions for the existence of linear invariant manifolds that correspond to certain modes of partial synchronization. It will be shown that such linear invariant manifolds can be identified by symmetries in the network. A network will show partial synchronization if a linear invariant manifold is attracting (for certain values of the coupling strength and time-delay). Conditions for the partial synchronization manifold to be attracting (in fact, to be asymptotically stable) are presented in Sec. V. To have partial synchronization, the conditions for the partial synchronization manifold to be attracting should not coincide with the conditions for the full synchronization manifold to be attracting. Sufficient conditions for full synchronization, hence necessary conditions for partial synchronization are given in Sec. VI. Section VII presents some examples of networks that show partial synchronization. Numerical simulations with Hindmarsh-Rose neurons support the results. Section VIII concludes the paper with a discussion of the results and possible extensions.

\section{PRELIMINARIES}

\section{A. Notation}

The symbol $\mathbb{R}$ stands for the real numbers $(-\infty, \infty), \mathbb{R}_{>0}\left(\mathbb{R}_{\geq 0}\right)$ denotes the set of positive (nonnegative) real numbers and $\mathbb{R}^{n}$ denotes the $n$-fold Cartesian product $\mathbb{R} \times \cdots \times \mathbb{R}$. The Euclidean norm in $\mathbb{R}^{n}$ is denoted by $|\cdot|,|x|^{2} \stackrel{\text { def }}{=} x^{\top} x$, where $x^{\top}$ denotes the transpose of $x$. The induced norm of a matrix $A \in \mathbb{R}^{n \times n}$, denoted by $\|A\|$, is defined as $\|A\| \stackrel{\text { def }}{=} \max _{x \in \mathbb{R}^{n},|x|=1}|A x|$. The $n \times n$ identity matrix is denoted by $I_{n}$. If no confusion will arise, we write $I$. The notation $\operatorname{col}\left(x_{1}, \ldots, x_{n}\right)$ denotes the column vector with entries $x_{1}, \ldots, x_{n}$. Here, $x_{i}$ may be scalars or column vectors. The notation $\operatorname{sp}\{\}$ stands for $\operatorname{span}\{\}$. The symbol $\otimes$ denotes the Kronecker product of two matrices, i.e., let $A \in \mathbb{R}^{n \times m}$ and $B \in \mathbb{R}^{p \times l}$, then the matrix $A \otimes B \in \mathbb{R}^{n p \times m l}$ is given as

$$
A \otimes B=\left(\begin{array}{cccc}
a_{11} B & a_{12} B & \ldots & a_{1 m} B \\
a_{21} B & a_{22} B & \ldots & a_{2 m} B \\
\vdots & \vdots & \ddots & \vdots \\
a_{n 1} B & a_{n 2} B & \ldots & a_{n m} B
\end{array}\right)
$$

where $a_{i j}$ denotes the $i j$ th entry of the matrix $A$. 
Let $\mathcal{X} \subset \mathbb{R}^{n}$ and $\mathcal{Y} \subset \mathbb{R}^{m}$. The space of continuous functions from $\mathcal{X}$ to $\mathcal{Y}$ that are (at least) $r \geq 0$ times continuously differentiable is denoted by $\mathcal{C}^{r}(\mathcal{X}, \mathcal{Y})$. For notational convenience, we will sometimes write $\mathcal{C}^{r}$ instead of $\mathcal{C}^{r}(\mathcal{X}, \mathcal{Y})$; and if $r=0$, we write $\mathcal{C}(\mathcal{X}, \mathcal{Y})$ instead of $\mathcal{C}^{0}(\mathcal{X}, \mathcal{Y})$. A function $V: \mathcal{D} \rightarrow \mathbb{R}_{\geq 0}, \mathcal{D} \subset \mathbb{R}^{n}$ contains 0 , is called positive (semi) definite, denoted by $V(\cdot)>0(V(\cdot) \geq 0)$, if $V(0)=0$ and $V(x)>0(V(x) \geq 0)$ for all $x \in \mathcal{D} \backslash\{0\}$. It is radially unbounded if $\mathcal{D}=\mathbb{R}^{n}$ and $|x| \rightarrow \infty$ imply $V(x) \rightarrow \infty$. If the quadratic form $x^{\top} P x$ with a symmetric matrix $P=P^{\top}$ is positive (semi) definite, then the matrix $P$ is positive (semi) definite, denoted by $P>0(P \geq 0)$.

Let $x_{t} \in \mathcal{C}\left([-\tau, 0], \mathbb{R}^{n}\right)$ be defined as $x(t+\theta), \theta \in[-\tau, 0]$. The norm of an element $x_{t} \in \mathcal{C}\left([-\tau, 0], \mathbb{R}^{n}\right)$ is denoted as $\left|x_{t}\right| \stackrel{\text { def }}{=} \sup _{\theta \in[-\tau, 0]}|x(t+\theta)|$. Note the abuse of notation, however, no confusion may arise.

Let $\mathcal{G}=\mathcal{G}(\mathcal{V}, \mathcal{E})$ be a graph with set of nodes $\mathcal{V}$ and set of $\operatorname{arcs} \mathcal{E}$. Given two nodes $i, j \in \mathcal{V}$, if there is a path of length 1 from $i$ to $j$, then $(i, j) \in \mathcal{E}$ and $i$ and $j$ are called adjacent. It will be assumed that the graph is

- simple, i.e., there are no self-loops, that is, for every $i \in \mathcal{V},(i, i) \notin \mathcal{E}$;

- strongly connected, i.e., for every two nodes $i, j \in \mathcal{V}$, there is a path between $i$ and $j$.

Note that we do not assume that $(i, j) \in \mathcal{E} \Rightarrow(j, i) \in \mathcal{E}$, that is, we allow the graph to be directed. Let $\mathcal{N}_{i}$ be the set of neighbors of node $i$ defined as $\mathcal{N}_{i}=\mathcal{N}_{i}(\mathcal{G})=\{j \in \mathcal{V} \mid(j, i) \in \mathcal{E}\}$.

\section{B. Semipassive systems and convergent systems}

Consider a system

$$
\begin{aligned}
& \dot{x}(t)=f(x(t), u(t)), \\
& y(t)=h(x(t)),
\end{aligned}
$$

with $x(t) \in \mathbb{R}^{n}$, input $u(t) \in \mathbb{R}^{m}$, output $y(t) \in \mathbb{R}^{m}$, and sufficiently smooth functions $f: \mathbb{R}^{n} \times \mathbb{R}^{m} \rightarrow \mathbb{R}^{n}, h: \mathbb{R}^{n} \rightarrow \mathbb{R}^{m}$.

Definition 1 (Semipassive systems ${ }^{53}$ ). System (2) is called $\mathcal{C}^{r}$-semipassive if there is a nonnegative function storage $V \in \mathcal{C}^{r}\left(\mathbb{R}^{n}, \mathbb{R}_{\geq 0}\right)$ and a function $H: \mathbb{R}^{n} \rightarrow \mathbb{R}$ such that

$$
V(x(t))-V\left(x\left(t_{0}\right)\right) \leq \int_{t_{0}}^{t} y^{\top}(s) u(s)-H(x(s)) \mathrm{d} s,
$$

with $H$ defined and nonnegative for all $x$ outside some ball $\mathcal{B}=\mathcal{B}(0, R) \subset \mathbb{R}^{n}$, i.e., for each $|x|>R, H(x) \geq \rho(|x|)$ with some continuous function $\rho: \mathbb{R}_{\geq 0} \rightarrow \mathbb{R}_{\geq 0}$. System (2) is called strictly $\mathcal{C}^{r}$-semipassive if it is $\mathcal{C}^{r}$-semipassive with $H$ defined and positive for all $x$ outside $\mathcal{B}$.

Remark 1. System (2) is $\mathcal{C}^{r}$-passive (strictly $\mathcal{C}^{r}$-passive) if it is $\mathcal{C}^{r}$-semipassive (strictly $\mathcal{C}^{r}$-semipassive) with $H$ being positive semidefinite (positive definite). (See Ref. 54 for the definition of a passive system.)

Remark 2. If $r \geq 1$, one can replace Eq. (3) by

$$
\dot{V}(x(t)) \leq y^{\top}(t) u(t)-H(x(t)) .
$$

Definition 2 (Convergent systems ${ }^{55,56}$ ). Consider the system

$$
\dot{x}(t)=f(x(t), u(t)),
$$

and suppose that $f$ is Lipschitz in $x$ and $u(t)$ is piecewise continuous in $t$ and takes values from a compact set $\mathcal{U} \subset \mathbb{R}^{m}$. The system is a convergent system if

(1) for each input $u(t)$ and every $x_{0}=x\left(t_{0}\right) \in \mathbb{R}^{n}$, all solutions $x\left(t ; t_{0}, u(t)\right)$ are defined and bounded on $t \in\left[t_{0}, \infty\right)$;

(2) for each input $u(t)$, there is an unique solution $x_{u}(t)$ that is defined and bounded on $t \in(-\infty, \infty)$ and is globally asymptotically stable.

The system is a uniformly convergent system (exponentially convergent system) if it is a convergent system and the unique limit solution $x_{u}(t)$ is uniformly globally asymptotically stable (globally exponentially stable).

Lemma 1 (Refs. 55 and 56). Consider the system (5) and suppose there is a positive definite matrix $P \in \mathbb{R}^{n \times n}$ such that the eigenvalues of the symmetric matrix

$$
\left[\frac{\partial f}{\partial x}(x, u)\right]^{\top} P+P\left[\frac{\partial f}{\partial x}(x, u)\right]
$$

are all negative and separated away from zero for all $x \in$ $\mathbb{R}^{n}$ and $u \in \mathcal{U}$. Then, the system is globally exponentially convergent.

\section{PROBLEM SETTING}

Consider $k$ systems

$$
\begin{aligned}
& \dot{x}_{i}(t)=f\left(x_{i}(t)\right)+B u_{i}(t), \\
& y_{i}(t)=C x_{i}(t), \quad i=1,2, \ldots, k,
\end{aligned}
$$

with $x_{i}(t) \in \mathbb{R}^{n}$, output $y_{i}(t) \in \mathbb{R}^{m}$, input $u_{i}(t) \in \mathbb{R}^{m}$, sufficiently smooth functions $f: \mathbb{R}^{n} \rightarrow \mathbb{R}^{n}$, and matrices $B, C$ of appropriate dimensions.

Let $\mathcal{G}$ be a simple and strongly connected graph and suppose that the systems (7) interact via diffusive time-delay coupling of the form

$$
u_{i}(t)=\sigma \sum_{j \in \mathcal{N}_{i}(\mathcal{G})} a_{i j}\left[y_{j}(t-\tau)-y_{i}(t)\right]
$$

or

$$
u_{i}(t)=\sigma \sum_{j \in \mathcal{N}_{i}(\mathcal{G})} a_{i j}\left[y_{j}(t-\tau)-y_{i}(t-\tau)\right],
$$

where constant $\sigma>0$ denotes the coupling strength, constant $\tau \geq 0$ is the time-delay and constant interconnection weights $a_{i j}>0$. Without loss of generality, we assume $\max _{i} \sum_{j \in \mathcal{N}_{i}(\mathcal{G})} a_{i j}=1$. We will refer to the former coupling as coupling $I$ while the latter coupling will be denoted as coupling II.

Consider the linear manifold

$$
\mathcal{M} \stackrel{\text { def }}{=}\left\{x \in \mathbb{R}^{k n} \mid x_{i}=x_{j} \quad \text { for all } i, j \in\{1,2, \ldots, k\}\right\} .
$$

Manifold $\mathcal{M}$ is called the full synchronization manifold, or simply synchronization manifold, for Eqs. (7) and (8) (or (7) 
and (9)) if it is invariant under the dynamics (7) and (8) (or (7) and (9)). Note that $\mathcal{M}$ is the synchronization manifold for the coupled systems (7) and (9) since the systems (7) are identical and the coupling functions (9) vanish on $\mathcal{M}$. To ensure that $\mathcal{M}$ is invariant under the dynamics (7) and (8), it will be assumed that $\sum_{j \in \mathcal{N}_{i}(\mathcal{G})} a_{i j}=1$ for all $i=1,2, \ldots, k$.

Definition 3. The systems (7) and (8) (or (7) and (9)) are said to fully synchronize, or simply synchronize, if the linear invariant synchronization manifold $\mathcal{M}$ contains an asymptotically stable subset.

Consider the linear manifold

$$
\mathcal{P} \stackrel{\text { def }}{=}\left\{x \in \mathbb{R}^{k n} \mid x_{i}=x_{j} \quad \text { for some } \quad \mathrm{i}, \mathrm{j} \in\{1,2, \ldots, k\}\right\},
$$

$n \leq \operatorname{dim} \mathcal{P} \leq(k-2) n$. The manifold $\mathcal{P}$ is called a partial synchronization manifold for Eqs. (7) and (8) (or Eqs. (7) and (9)) if it is invariant under the dynamics (7) and (8) (or (7) and (9)). Note that multiple partial synchronization manifolds may exist and that the full synchronization manifold is the intersection of all partial synchronization manifolds.

Definition 4. The systems (7) and (8) (or (7) and (9)) are said to partially synchronize if the linear invariant partial synchronization manifold $\mathcal{P}$ contains an asymptotically stable subset.

This definition states, roughly speaking, that a network exhibits partial synchronization if the states of at least two but not all systems asymptotically match. The remainder of this paper is devoted to identifying partial synchronization manifolds and give conditions for these manifolds to contain an asymptotically stable subset. But before doing so, we first introduce some matrices that define the coupling structure and we present a result from Ref. 39 regarding boundedness of solutions of the coupled systems (7) and (8) (or (7) and (9)).

Let the $i j$ th entry of the matrix $A$ be $a_{i j}$ if $j \in \mathcal{N}_{i}(\mathcal{G})$ and 0 otherwise. Hence, the matrix $A$ is a weighted adjacency matrix. It follows from the assumption that $\mathcal{G}$ is strongly connected that $A$ is irreducible. Let $D \in \mathbb{R}^{k \times k}$ be a diagonal matrix with $d_{i}=\sum_{j \in \mathcal{N}_{i}(\mathcal{G})} a_{i j}$ as $i$ th entry. Note that for coupling I, we have $D=I$ by assumption. (Although not strictly necessary, it will still be assumed that, for each $i$, $\sum_{j \in \mathcal{N}_{i}(\mathcal{G})} a_{i j}=1$.) Let $L=D-A$ be the weighted Laplacian matrix. In the remainder of this paper, we simply write adjacency matrix and Laplacian matrix instead of weighted adjacency matrix and weighted Lapacian matrix, respectively.

Lemma 2. Let $\mathcal{G}$ be a simple strongly connected graph. Suppose that

- each system (7) is strictly $\mathcal{C}^{1}$-semipassive with a radially unbounded storage function and there is a constant $\epsilon>0$ such that $H(x)-\epsilon|x|^{2}>0$ for sufficiently large $|x|$. Then, the solutions of the coupled systems (7) and (8) are ultimately bounded;

- each system (7) is strictly $\mathcal{C}^{1}$-semipassive with a radially unbounded storage function and there is a constant $\epsilon>0$ such that $H(x)-\epsilon|x|^{2}-2 \sigma|h(x)|^{2}>0$ for sufficiently large $|x|$ and all $\sigma \in\left[0, \sigma_{\max }\right], \sigma_{\max }>0$. Then, the solutions of the coupled systems (7) and (9) are ultimately bounded.
The proof of Lemma 2 is based on the construction of a Lyapunov functional of the form

$$
\begin{aligned}
& \nu_{1} V\left(x_{1}(t)\right)+\cdots+\nu_{k} V\left(x_{k}(t)\right) \\
& +\frac{\sigma}{2} \sum_{i=1}^{k} \sum_{j \in \mathcal{N}_{i}(\mathcal{G})} \nu_{i} a_{i j} \int_{-\tau}^{0}\left|y_{j}(t+s)\right|^{2} \mathrm{~d} s,
\end{aligned}
$$

where $V$ are the storage functions from the semipassivity assumption and $\nu_{i}$ are the entries of the left eigenvector corresponding to the simple zero eigenvalue of the Laplacian matrix $L$. Note that $L$ is singular by construction and the assumption that $\mathcal{G}$ is strongly connected implies that the zero eigenvalue is simple. Using the Perron-Frobenius theorem, one can show that all $\nu_{i}>0$. One can show that the derivative of this functional along solutions is negative for sufficiently large $|x|=\left|\operatorname{col}\left(x_{1}, \ldots, x_{k}\right)\right|$. Then, a straightforward application of Ref. 57, Theorem 4.2.10, pp. 272, implies the result. See, Ref. 39 for further details.

It has to be remarked that for systems that interact via coupling type II there is a limit on the coupling strength for which boundedness can be guaranteed.

\section{SYMMETRIES AND INVARIANT MANIFOLDS}

As mentioned in the introduction, we extend the ideas presented in Refs. 44 and 45 for identification of partial synchronization modes to the case of general time-delay diffusive coupling. The idea is simply to search for rearrangements of the nodes that leave the network essentially unchanged. That is, the structure of the network is preserved after simultaneous swapping of (some of) the nodes of the network. Such a rearrangement can be conveniently expressed using permutation matrices. Let $\Pi \in \mathbb{R}^{k \times k}$ be a permutation matrix, then it is easy to see that the network is invariant under rearrangement induced by $\Pi$ if $\Pi A \Pi^{\top}=A$, i.e., $A$ and $\Pi$ commute. Moreover, in that case, the set $\operatorname{ker}\left(I_{k n}-\Pi \otimes I_{n}\right)$ will define a linear invariant manifold of the form (11) for the coupled systems. A formal and more general statement for systems interacting via coupling I is presented in the following lemma.

Lemma 3. Consider a network with $k$ systems (7) that interact via coupling $I$ (8). Let $\Pi \in \mathbb{R}^{k \times k}$ be permutation matrix and $A \in \mathbb{R}^{k \times k}$ be the adjacency matrix. If there is a solution $X \in \mathbb{R}^{k \times k}$ to the matrix equation

$$
(I-\Pi) A=X(I-\Pi)
$$

then the set $\operatorname{ker}\left(I_{k n}-\Pi \otimes I_{n}\right)$ defines a linear invariant manifold for the coupled systems (7) and (8).

Proof. Let $x(t)=\operatorname{col}\left(x_{1}(t), \ldots, x_{k}(t)\right)$ and introduce new coordinates $w(t)=\left(I_{k n}-\Pi \otimes I_{n}\right) x(t)$. Then,

$$
\begin{aligned}
\dot{w}(t)= & \left(I_{k n}-\Pi \otimes I_{n}\right)[F(x(t))-\sigma(I \otimes B C) x(t) \\
& +\sigma(A \otimes B C) x(t-\tau)],
\end{aligned}
$$

with $F(x(t)) \stackrel{\text { def }}{=} \operatorname{col}\left(f\left(x_{1}(t)\right), \ldots, f\left(x_{k}(t)\right)\right)$. Assume that the initial history $\phi \stackrel{\text { def }}{=} x_{t_{0}} \in \mathcal{C}\left([-\tau, 0], \mathbb{R}^{k n}\right)$ is such that 
$\left(I_{k n}-\Pi \otimes I_{n}\right) \phi \equiv 0$. It follows that the set $\operatorname{ker}\left(I_{k n}-\Pi \otimes I_{n}\right)$ is invariant under the dynamics if the assumption that $\phi$ constrained to this set implies $\dot{w}(t) \equiv 0$. Consider the right hand side of Eq. (12) and observe that, for every $x\left(t^{*}\right) \in$ ker $\left(I_{k n}-\Pi \otimes I_{n}\right) \Longleftrightarrow w\left(t^{*}\right)=0, t^{*} \in[-\tau, \infty)$,

- $\left(I_{k n}-\Pi \otimes I_{n}\right) F\left(x\left(t^{*}\right)\right)=F\left(x\left(t^{*}\right)\right)-F\left(\left(\Pi \otimes I_{n}\right) x\left(t^{*}\right)\right)=0$;

- $\left(I_{k n}-\Pi \otimes I_{n}\right)(I \otimes B C) x\left(t^{*}\right)=(I \otimes B C) w\left(t^{*}\right)=0 ;$

- $\left(I_{k n}-\Pi \otimes I_{n}\right)(A \otimes B C) x\left(t^{*}\right)=(X \otimes B C) w\left(t^{*}\right)=0$.

In the latter equality, we used $(I-\Pi) A=X(I-\Pi)$. Hence, $\dot{w}(t)=0$, which proves that $\operatorname{ker}\left(I_{k n}-\Pi \otimes I_{n}\right)$ is invariant under the dynamics.

A similar result can be obtained for systems that interact via coupling II.

Lemma 4. Consider a network with $k$ systems (7) that interact via coupling II (9). Let $\Pi \in \mathbb{R}^{k \times k}$ be permutation matrix and $L \in \mathbb{R}^{k \times k}$ be the Laplacian matrix. If there is a solution $X \in \mathbb{R}^{k \times k}$ to the matrix equation

$$
(I-\Pi) L=X(I-\Pi)
$$

then the set $\operatorname{ker}\left(I_{k n}-\Pi \otimes I_{n}\right)$ defines a linear invariant manifold for the coupled systems (7) and (9).

The proof follows with minor modifications from the proof of Lemma 3 and will, therefore, be omitted.

Remark 3. Obviously, if $A$ and $\Pi$ commute (Lemma 3) or $L$ and $\Pi$ commute (Lemma 4 ), then $X=A$ or $X=L$, respectively.

The example below shows how the results of Lemma 3 (and Lemma 4) can be applied.

Example 1. Consider the network shown in Figure 1(a). The adjacency matrix for this network is

$$
A=\frac{1}{3}\left(\begin{array}{llll}
0 & 1 & 0 & 2 \\
1 & 0 & 2 & 0 \\
0 & 2 & 0 & 1 \\
2 & 0 & 1 & 0
\end{array}\right)
$$

The matrix A commutes with the following permutation matrices

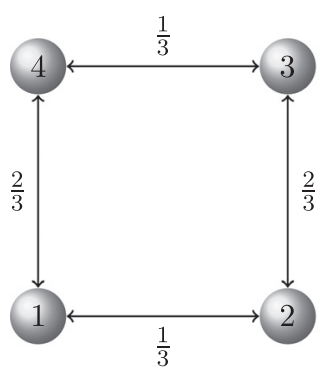

(a)

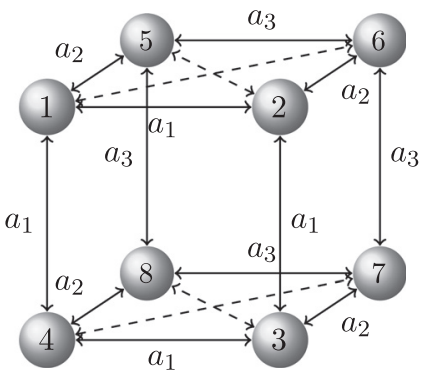

(b)
FIG. 1. The networks of (a): example 1 and (b): example 2. (Dashed lines have weight $a_{4}$.)

$$
\begin{aligned}
\Pi_{1} & =\left(\begin{array}{llll}
0 & 1 & 0 & 0 \\
1 & 0 & 0 & 0 \\
0 & 0 & 0 & 1 \\
0 & 0 & 1 & 0
\end{array}\right), \Pi_{2}=\left(\begin{array}{llll}
0 & 0 & 1 & 0 \\
0 & 0 & 0 & 1 \\
1 & 0 & 0 & 0 \\
0 & 1 & 0 & 0
\end{array}\right), \\
\Pi_{3} & =\left(\begin{array}{llll}
0 & 0 & 0 & 1 \\
0 & 0 & 1 & 0 \\
0 & 1 & 0 & 0 \\
1 & 0 & 0 & 0
\end{array}\right) .
\end{aligned}
$$

Then, each set $\operatorname{ker}\left(I_{4 n}-\Pi_{1} \otimes I_{n}\right), \operatorname{ker}\left(I_{4 n}-\Pi_{2} \otimes I_{n}\right)$, and $\operatorname{ker}\left(I_{4 n}-\Pi_{3} \otimes I_{n}\right)$ defines a linear invariant manifold for the coupled systems (7) and (8) or (7) and (9). Observe that, for instance, $\operatorname{ker}\left(I_{4 n}-\Pi_{1} \otimes I_{n}\right) \cap \operatorname{ker}\left(I_{4 n}-\Pi_{2} \otimes I_{n}\right)$ defines the full synchronization manifold.

Example 2. (See also Ref. 45.) Consider the network shown in Figure 1(b). The adjacency matrix of this network is

$$
A=\left(\begin{array}{cccccccc}
0 & -a_{1} & 0 & -a_{1} & -a_{2} & -a_{4} & 0 & 0 \\
-a_{1} & 0 & -a_{1} & 0 & -a_{4} & -a_{2} & 0 & 0 \\
0 & -a_{1} & 0 & -a_{1} & 0 & 0 & -a_{2} & -a_{4} \\
-a_{1} & 0 & -a_{1} & 0 & 0 & 0 & -a_{4} & -a_{2} \\
-a_{2} & -a_{4} & 0 & 0 & 0 & -a_{3} & 0 & -a_{3} \\
-a_{4} & -a_{2} & 0 & 0 & -a_{3} & 0 & -a_{3} & 0 \\
0 & 0 & -a_{2} & -a_{4} & 0 & -a_{3} & 0 & -a_{3} \\
0 & 0 & -a_{4} & -a_{2} & -a_{3} & 0 & -a_{3} & 0
\end{array}\right) .
$$

Let $a_{1}=a_{3}=\frac{4}{11}$ and $a_{2}=\frac{1}{11} a_{4}=\frac{2}{11}$, then the permutation matrix

$$
\Pi=\left(\begin{array}{llllllll}
0 & 1 & 0 & 0 & 0 & 0 & 0 & 0 \\
0 & 0 & 1 & 0 & 0 & 0 & 0 & 0 \\
0 & 0 & 0 & 1 & 0 & 0 & 0 & 0 \\
1 & 0 & 0 & 0 & 0 & 0 & 0 & 0 \\
0 & 0 & 0 & 0 & 0 & 1 & 0 & 0 \\
0 & 0 & 0 & 0 & 0 & 0 & 1 & 0 \\
0 & 0 & 0 & 0 & 0 & 0 & 0 & 1 \\
0 & 0 & 0 & 0 & 1 & 0 & 0 & 0
\end{array}\right)
$$

does not commute with $L=D-A$ but there exists a $X$ that solves the matrix equation $(I-\Pi) L=X(I-\Pi)$. See, Sec.VII B for details. Thus, the set $\operatorname{ker}\left(I_{8 n}-\Pi \otimes I_{n}\right)$ defines a linear invariant manifold for the coupled systems (7) and (8) or (7) and (9).

\section{PARTIAL SYNCHRONIZATION}

In Sec. V, conditions are presented for the existence of linear invariant manifolds. For partial synchronization, we require these manifolds to contain an asymptotically stable subset. In this section, sufficient conditions will be presented for the linear invariant manifolds to contain an asymptotically stable subset.

Assume that the matrix $C B$ is similar to a positive definite matrix. Then, there is a smooth change of coordinates $x_{i} \longmapsto\left(z_{i}, y_{i}\right)$ that transforms the systems (7) into the following form:

$$
\dot{z}_{i}(t)=q\left(z_{i}(t), y_{i}(t)\right)
$$




$$
\dot{y}_{i}(t)=a\left(y_{i}(t), z_{i}(t)\right)+C B u_{i}(t)
$$

with $z_{i}(t) \in \mathbb{R}^{p}, p=n-m, y_{i}(t) \in \mathbb{R}^{m}$ and sufficiently smooth functions $q: \mathbb{R}^{p} \times \mathbb{R}^{m} \rightarrow \mathbb{R}^{p}, a: \mathbb{R}^{m} \times \mathbb{R}^{p} \rightarrow \mathbb{R}^{m}$. See, for instance, Ref. 44 for details. For notational convenience and we assume $C B=I$ and $x_{i}(t)=\operatorname{col}\left(z_{i}(t), y_{i}(t)\right)$. (Results for the general case with $C B$ being positive definite can easily be derived.)

We would like to add some remarks on the structure of systems (13). The inputs $u_{i}(t)$ appear only in the output dynamics, i.e., $y_{i}$-dynamics. This type of systems is known in the control systems community as relative degree one systems. If we assume that the systems in original coordinates, i.e., systems (7), are strictly semipassive, then it is likely that the systems have relative degree one (and hence, after a suitable change of coordinates, take the structure (13)). This follows from classical results that strictly passive systems (recall that a system is strictly passive if it satisfies the definition of a semipassive system with the function $H$ being positive definite, see, Remark 1) with a positive definite $\mathcal{C}^{2}$ storage function should be relative degree one systems, cf. (Ref. 58), and the fact that a strictly semipassive systems behaves as a strictly passive system for sufficiently large $|x|$. Since there is no direct influence of the inputs $u_{i}(t)$ on the "internal" $z_{i}$-dynamics, we have to impose some conditions on these internal dynamics that guarantee $\lim _{t \rightarrow \infty}\left(z_{i}(t)\right.$ $\left.-z_{j}(t)\right)=0$ provided that $\lim _{t \rightarrow \infty}\left(y_{i}(t)-y_{j}(t)\right)=0$ for some (all) pairs $(i, j), i, j \in\{1,2, \ldots, k\}$. Obviously, this is true if the internal dynamics are convergent (see, Sec. II). In Ref. 39 , it is shown that assuming that the systems (13) (or systems (7) before the change of coordinates) satisfy the conditions of Lemma 2 and have convergent internal dynamics guarantees the existence of positive constants $\bar{\sigma}$ and $\bar{\gamma}$ such that if $\sigma>\bar{\sigma}$ and $\sigma \tau<\bar{\gamma}$, then the synchronization manifold $\mathcal{M}$ contains a globally asymptotically stable subset. In other words, strictly semipassive systems with convergent internal dynamics on a simple strongly connected graph are guaranteed to synchronize provided that the coupling is sufficiently strong and the product of the coupling strength and timedelay is sufficiently small. See, Figure 2 . It has to be

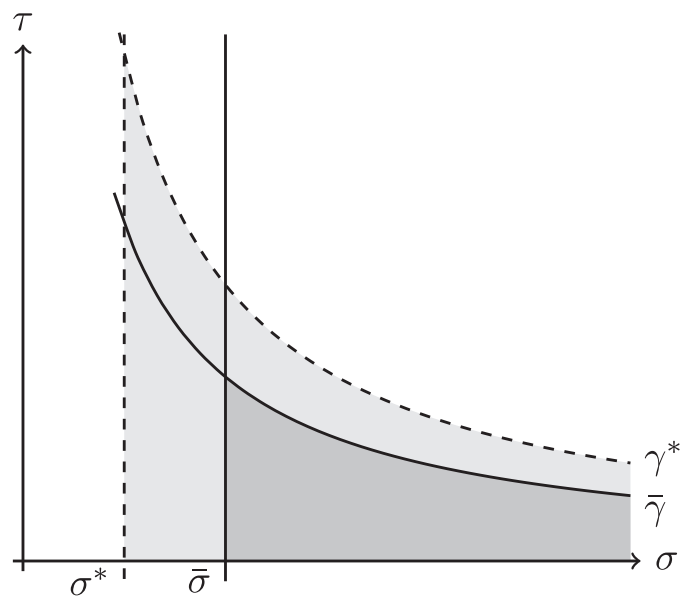

FIG. 2. Guaranteed asymptotic stability of a subset of the set $\operatorname{ker}\left(I_{k n}-\Pi \otimes\right.$ $I_{n}$ ) (light shaded areas) and the full synchronization manifold $\mathcal{M}$ (dark shaded area) for systems that interact with coupling I under the assumption that $0<\sigma^{*}<\bar{\sigma}$ and $\gamma^{*}>\bar{\gamma}>0$. remarked that the values of $\bar{\sigma}$ and $\bar{\gamma}$ depend on the type of coupling (coupling I or coupling II), the type of systems and the topology of the network. We will show that the assumptions that the systems are semipassive and have internal convergent dynamics are also sufficient to guarantee the existence of an asymptotically stable subset of the partial synchronization manifolds $\mathcal{P}$. For systems that interact via coupling I, we have the following result.

Theorem 5. Consider $k$ coupled systems (13) and (8) with $\mathcal{G}$ being simple and strongly connected. Suppose that the conditions of Lemma 2 and Lemma 3 are satisfied for some matrix $X$ and permutation matrix $\Pi$. Assume in addition that

- there is a constant $c^{*}>0$ such that

$$
\vartheta^{\top}(I-\Pi)^{\top}\left(I_{k}-\frac{1}{2}\left(X^{\top}+X\right)\right)(I-\Pi) \vartheta \geq c^{*}|(I-\Pi) \vartheta|^{2}
$$

for every $\vartheta \in \mathbb{R}^{k}$;

- there is a symmetric matrix $P \in \mathbb{R}^{k \times k}$ such that the eigenvalues of the symmetric matrix

$$
\left[\frac{\partial q}{\partial z_{i}}\left(z_{i}, y_{i}\right)\right]^{\top} P+P\left[\frac{\partial q}{\partial z_{i}}\left(z_{i}, y_{i}\right)\right]
$$

are negative and bounded away from zero for all $z_{i} \in \mathbb{R}^{p}$ and $y_{i} \in \mathbb{R}^{m}$.

Then, there exist positive constants $\sigma^{*}$ and $\gamma^{*}$ such that if $\sigma>\sigma^{*}$ and $\sigma \tau<\gamma^{*}$, then the set $\operatorname{ker}\left(I_{k n}-\Pi \otimes I_{n}\right)$ contains a globally asymptotically stable subset.

For systems which interact via coupling II, we have a similar result.

Theorem 6. Consider $k$ coupled systems (13) and (9) with $\mathcal{G}$ being simple and strongly connected. Suppose that the conditions of Lemma 2 and Lemma 4 are satisfied for some matrix $X$ and permutation matrix $\Pi$. Assume in addition that

- there is a constant $c^{\prime}>0$ such that

$$
\frac{1}{2} \vartheta^{\top}(I-\Pi)^{\top}\left(X^{\top}+X\right)(I-\Pi) \vartheta \geq c^{\prime}|(I-\Pi) \vartheta|^{2}
$$

for every $\vartheta \in \mathbb{R}^{k}$;

- there is a symmetric matrix $P \in \mathbb{R}^{k \times k}$ such that the eigenvalues of the symmetric matrix

$$
\left[\frac{\partial q}{\partial z_{i}}\left(z_{i}, y_{i}\right)\right]^{\top} P+P\left[\frac{\partial q}{\partial z_{i}}\left(z_{i}, y_{i}\right)\right]
$$

are negative and bounded away from zero for all $z_{i} \in \mathbb{R}^{p}$ and $y_{i} \in \mathbb{R}^{m}$.

Then, there exist positive constants $\sigma^{\prime}$ and $\gamma^{\prime}$ such that if $\sigma^{\prime}<$ $\sigma \leq \sigma_{\max }$ (with $\sigma_{\max }$ being the maximal coupling strength for which boundedness of solutions is guaranteed) and $\sigma \tau<\gamma^{\prime}$, then the set $\operatorname{ker}\left(I_{k n}-\Pi \otimes I_{n}\right)$ contains a globally asymptotically stable subset.

Remark 4. Theorem 5 and Theorem 6 remain valid without the assumptions of Lemma 2 (systems are not strictly semipassive) if the solutions of the whole network are ultimately bounded. 
The proof of both Theorem 5 and Theorem 6 can be found in the Appendix in which also the estimates of the constants $\sigma^{*}$ and $\gamma^{*}, \sigma^{\prime}$ and $\gamma^{\prime}$ respectively, are provided.

It has to be remarked that the values of the constants $\sigma^{*}$ and $\gamma^{*}$ (or $\sigma^{\prime}$ and $\gamma^{\prime}$ ) depend on the type of systems in the network and the coupling structure and, in particular, on the matrices $\Pi$ and $X$. (See, the proofs of the theorems.)

\section{FULL SYNCHRONIZATION AND NECESSARY CONDITIONS FOR PARTIAL SYNCHRONIZATION}

As mentioned before, multiple partial synchronization manifolds might coexist and also the conditions for such manifolds to be stable might coincide. The case for which all partial synchronization manifolds are stable coincides the fully synchronized state. It follows that to observe partial synchronization, it is necessary that the values of the coupling strength and time-delay for which the partial manifold is stable do not coincide with those for which the full synchronization manifold is stable. However, we already know that full synchronization will happen in networks of systems that satisfy the assumptions of Theorems 5 and 6.

Theorem 7. (Ref. 39). Consider $k$ coupled systems (13) and (8) or (13) and (9) with $\mathcal{G}$ being simple and strongly connected. Suppose that the conditions of Lemma 2 are satisfied. Assume in addition that there is a symmetric matrix $P \in$ $\mathbb{R}^{k \times k}$ such that the eigenvalues of the symmetric matrix

$$
\left[\frac{\partial q}{\partial z_{i}}\left(z_{i}, y_{i}\right)\right]^{\top} P+P\left[\frac{\partial q}{\partial z_{i}}\left(z_{i}, y_{i}\right)\right]
$$

are negative and bounded away from zero for all $z_{i} \in \mathbb{R}^{p}$ and $y_{i} \in \mathbb{R}^{m}$. Then, there exist positive constants $\bar{\sigma}$ and $\bar{\gamma}$ such that if $\sigma>\bar{\sigma}$ and $\sigma \tau<\bar{\gamma}$ then the synchronization manifold $\mathcal{M}$ contains a globally asymptotically stable subset.

It follows that to have partial synchronization, it is necessary that $\sigma^{*}<\bar{\sigma}$ and/or $\gamma^{*}>\bar{\gamma}$ for coupled systems (8) and (13), or $\sigma^{\prime}<\bar{\sigma}$ and/or $\gamma^{\prime}>\bar{\gamma}$ for coupled systems (9) and (13). An example where partial synchronization can be observed is schematically depicted in Figure 2.

For some network structures, it is possible to derive necessary conditions for not having asymptotic stability of a subset of the partial synchronization manifold and a subset of the full synchronization for the same values of $\sigma$ and $\tau$. For systems that interact via coupling I, we have the following result.

Corollary 8. Consider the coupled systems (13) and (8) and let the conditions of Theorem 5 be satisfied. Assume in addition that $\Pi$ and $A$ commute and that the eigenvalues of $A$ are real with right eigenvectors that are linearly independent. Then, the eigenvalues of $A$ can be ordered as

$$
-1 \leq \lambda_{1}(A) \leq \lambda_{2}(A) \leq \ldots \leq \lambda_{k-1}(A)<\lambda_{k}(A)=1 .
$$

Let $\mu\left(\lambda_{i}(A)\right)$ be a right eigenvector corresponding to eigenvalue $\lambda_{i}(A)$ and let

- $\underline{\lambda}(A)$ the eigenvalue of $A$ with largest absolute value with the restriction that $\mu(\underline{\lambda}(A)) \in$ range $(I-\Pi)$;

- $\bar{\lambda}(A)$ the largest eigenvalue of $A$ with the restriction that $\mu(\bar{\lambda}(A)) \in \operatorname{range}(I-\Pi)$.
Suppose that the eigenvectors in range $(I-\Pi)$ are mutually orthogonal. Then,

- $\sigma^{*}<\bar{\sigma}$ only if $\bar{\lambda}(A)<\lambda_{k-1}(A)$;

- $\gamma^{*}>\bar{\gamma}$ only if $\underline{\lambda}(A)<\max \left\{\left|\lambda_{1}(A)\right|,\left|\lambda_{k-1}(A)\right|\right\}$.

Proof. All eigenvalues of $A$ are real by assumption. Moreover, we always assume that all rows of $A$ sum up to 1 and $A$ is irreducible since $\mathcal{G}$ is strongly connected. Then, the Perron-Frobenius theorem together with Gerschgorin's theorem imply that $\lambda_{k}=1$ and all other eigenvalues are smaller than $\lambda_{k}$, and $\left|\lambda_{j}\right| \leq 1$ for every $j=1,2, \ldots, k$. Let $A U=U \Lambda$ with $\Lambda$ being a diagonal matrix with the eigenvalues of $A$ as entries and the columns of $U$ are corresponding eigenvalues. Let $d$ be the number of eigenvectors in range $(I-\Pi)$. Then, without loss of generality, we assume that the first $d$ columns of $U$ are eigenvectors in range $(I-\Pi)$ and, obviously, the first $d$ diagonal entries of $\Lambda$ will be the corresponding eigenvalues. Because $A$ and $\Pi$ commute, we have $(I-\Pi) A$ $=X(I-\Pi)$ with $X=A$. We will now show that $X=\tilde{U} \Lambda U^{-1}$ with $\tilde{U}=\left(\begin{array}{lll}u_{1} \ldots u_{d} & 0 \ldots 0\end{array}\right)$ also solves $(I-\Pi) A=X$ $(I-\Pi)$. Note that $\tilde{U}=U \Delta_{1}$ with $\Delta_{1}$ a diagonal matrix where the first $d$ entries equal 1 and all other entries are 0 . Because the first $d$ columns of $U$ are eigenvectors in range $(I-\Pi)$, we can write $(I-\Pi)=\tilde{U} \Delta_{2}$ for some $\Delta_{2} \in \mathbb{R}^{k \times k}$. Then,

$$
(I-\Pi) A=A(I-\Pi)=A \tilde{U} \Delta_{2}=A U \Delta_{1} \Delta_{2}=U \Lambda \Delta_{1} \Delta_{2} .
$$

This also gives

$X(I-\Pi)=\tilde{U} \Lambda U^{-1}(I-\Pi)=\tilde{U} U^{-1} A(I-\Pi)=\tilde{U} \Lambda \Delta_{1} \Delta_{2}$.

Thus, $X=\tilde{U} \Lambda U^{-1}$ solves $(I-\Pi) A=X(I-\Pi)$ if $(U-\tilde{U})$ $\Lambda \Delta_{1} \Delta_{2}=0$. This is clearly true (for every $\Delta_{2}$ ) since $U-\tilde{U}=\left(\begin{array}{ll}\mathbf{0}_{k \times d} & U^{*}\end{array}\right)$, with matrix $U^{*} \in \mathbb{R}^{k \times(k-d)}$ whose columns consist of eigenvectors $u_{d+1}, \ldots, u_{k}$, and $\Lambda \Delta_{1}$ $=\operatorname{diag}\left(\left[\mathbf{1}_{d} \mathbf{0}_{(k-d) \times 1}\right]\right)$. Here, $\mathbf{1}_{d}$ the $d$-dimensional vector with all entries equal 1 , and $\mathbf{0}_{d_{1} \times d_{2}}$ denotes the $d_{1} \times d_{2}$-dimensional zero matrix. It follows that, under the assumptions of the corollary,

$$
\vartheta^{\top}(I-\Pi)^{\top}\left(I-\frac{1}{2}\left(X^{\top}+X\right)\right)(I-\Pi) \vartheta \geq c^{*}|(I-\Pi) \vartheta|^{2}
$$

for every $\vartheta \in \mathbb{R}^{k}$ with $c^{*}=(1-\bar{\lambda}(A))$. In addition, $\|X\| \leq \underline{\lambda}(A)$. Recall that we will have full synchronization if all partial synchronization modes are stable at the same time. We conclude that picking $\underline{\lambda}(A)=\max \left\{\left|\lambda_{1}(A)\right|,\left|\lambda_{k-1}(A)\right|\right\}$ and $\bar{\lambda}(A)=\lambda_{k-1}(A)$ are sufficient to have full synchronization. Hence to have partial synchronization, it is necessary that $\bar{\lambda}(A)<\lambda_{k-1}(A)$ and/or $\underline{\lambda}(A)<\max \left\{\left|\lambda_{1}(A)\right|,\left|\lambda_{k-1}(A)\right|\right\}$. The remaining part of the proof follows now directly from the proof of Theorem 5 .

For systems that interact via coupling II, we have the following result.

Corollary 9. Consider the coupled systems (13) and (9) and let the conditions of Theorem 6 be satisfied. Assume in addition that $\Pi$ and $L$ commute and that the eigenvalues of $L$ are real with right eigenvectors that are linearly independent. Then, the eigenvalues of L are 


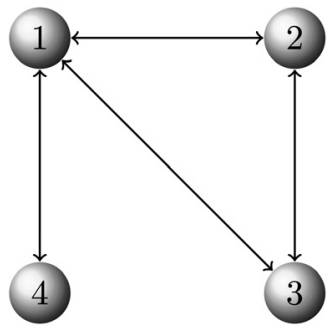

FIG. 3. The network of example 3

$$
0=\lambda_{1}(L)<\lambda_{2}(L) \leq \ldots \leq \lambda_{k-1}(L) \leq \lambda_{k}(L)
$$

Let $\mu\left(\lambda_{i}(L)\right)$ be a right eigenvector corresponding to eigenvalue $\lambda_{i}(L)$ and let

- $\underline{\lambda}(L)$ the smallest nonzero eigenvalue of $L$ with the restriction that $\mu(\underline{\lambda}(L)) \in \operatorname{range}(I-\Pi)$;

- $\bar{\lambda}(L)$ the largest eigenvalue of $L$ with the restriction that $\mu(\bar{\lambda}(L)) \in \operatorname{range}(I-\Pi)$.

Suppose that the eigenvectors in range $(I-\Pi)$ are mutually orthogonal. Then,

- $\sigma^{\prime}<\bar{\sigma}$ only if $\underline{\lambda}(L)>\lambda_{2}(L)$;

- $\gamma^{\prime}>\bar{\gamma}$ only if $\bar{\lambda}(L)<\lambda_{k}(L)$.

The proof of the latter corollary can easily be deducted from the proof of Theorem 6 and Corollary 8 and will be omitted.

Example 3. Consider the network shown in Figure 3. The Laplacian matrix is

$$
L=\frac{1}{3}\left(\begin{array}{cccc}
3 & -1 & -1 & -1 \\
-1 & 2 & -1 & 0 \\
-1 & -1 & 2 & 0 \\
-1 & 0 & 0 & 1
\end{array}\right)
$$

which has eigenvalues

$$
\lambda_{1}=0, \quad \lambda_{2}=\frac{1}{3}, \quad \lambda_{3}=1, \quad \lambda_{4}=\frac{4}{3},
$$

with eigenvectors $\mu_{i}=\mu\left(\lambda_{i}\right)$

$$
\begin{aligned}
& \mu_{1} \in \operatorname{sp}\left\{\left(\begin{array}{l}
1 \\
1 \\
1 \\
1
\end{array}\right), \quad \mu_{2} \in \operatorname{sp}\left\{\left(\begin{array}{c}
1 \\
-1 \\
-1 \\
2
\end{array}\right)\right\},\right. \\
& \mu_{3} \in \operatorname{sp}\left\{\left(\begin{array}{c}
0 \\
1 \\
-1 \\
0
\end{array}\right)\right\}, \quad \mu_{4} \in \operatorname{sp}\left\{\left(\begin{array}{c}
3 \\
-1 \\
-1 \\
-1
\end{array}\right)\right\} .
\end{aligned}
$$

It is easy to see that $L$ commutes with the permutation matrix

$$
\Pi=\left(\begin{array}{llll}
1 & 0 & 0 & 0 \\
0 & 0 & 1 & 0 \\
0 & 1 & 0 & 0 \\
0 & 0 & 0 & 1
\end{array}\right)
$$

and the only eigenvector in range $(I-\Pi)$ is $\mu_{3}$. Using Corollary 9, we conclude that partial synchronization of systems 2 and 3 is guaranteed for values of the coupling strength and time-delay other than those for which full synchronization is guaranteed, i.e., the conditions for full synchronization and partial synchronization do not necessarily coincide. The expected (partial) synchronization diagram is as the one shown in Figure 2.

\section{EXAMPLES}

In this section, we present examples of partial synchronization in networks of Hindmarsh-Rose neurons. Consider $k$ Hindmarsh-Rose neurons ${ }^{59}$

$$
\begin{aligned}
& \dot{z}_{1, i}(t)=0.005\left(4\left(y_{i}(t)+1.618\right)-z_{1, i}(t)\right) \\
& \left.\dot{z}_{2, i}(t)=1-5 y_{i}^{2}(t)-z_{2, i}(t)\right) \\
& \dot{y}_{i}(t)=-y_{i}^{3}(t)+3 y_{i}^{2}(t)+3.25+z_{2, i}(t)-z_{1, i}(t)+u_{i}(t) .
\end{aligned}
$$

Here, $y_{i}(t)$ denotes the membrane potential of the $i$ th neuron, which is also its output. The internal variables $z_{1, i}, z_{2, i}$ are related to ionic currents. It is shown in Ref. 60 that the Hindmarsh-Rose neuron satisfies the conditions in Lemma 2, i.e., the model has the strict semipassivity property. Moreover, its internal dynamics are convergent. The latter can be easily verified using Lemma 1 with $P=I$. Thus, a network with these neurons, which interact either via coupling I or coupling II will synchronize provided that the coupling is sufficiently strong, and the product of the coupling and timedelay is sufficiently small. Moreover, our theory suggests that certain networks might exhibit partial synchronization.

\section{A. Network of example 1}

Consider the network of example 1 and recall that the permutation matrices

$$
\begin{aligned}
\Pi_{1} & =\left(\begin{array}{llll}
0 & 1 & 0 & 0 \\
1 & 0 & 0 & 0 \\
0 & 0 & 0 & 1 \\
0 & 0 & 1 & 0
\end{array}\right), \quad \Pi_{2}=\left(\begin{array}{llll}
0 & 0 & 1 & 0 \\
0 & 0 & 0 & 1 \\
1 & 0 & 0 & 0 \\
0 & 1 & 0 & 0
\end{array}\right), \\
\Pi_{3} & =\left(\begin{array}{llll}
0 & 0 & 0 & 1 \\
0 & 0 & 1 & 0 \\
0 & 1 & 0 & 0 \\
1 & 0 & 0 & 0
\end{array}\right),
\end{aligned}
$$

commute with the adjacency matrix 


$$
A=\frac{1}{3}\left(\begin{array}{llll}
0 & 1 & 0 & 2 \\
1 & 0 & 2 & 0 \\
0 & 2 & 0 & 1 \\
2 & 0 & 1 & 0
\end{array}\right)
$$

Let, $\lambda_{i}$ be an eigenvalue of $A$ with eigenvector $\mu_{i}$. A straightforward computation shows that $A$ has eigenvalues

$$
\lambda_{1}=-1, \quad \lambda_{2}=-\frac{1}{3}, \quad \lambda_{3}=\frac{1}{3}, \quad \lambda_{4}=1,
$$

with eigenvectors

$$
\begin{aligned}
& \mu_{1} \in \operatorname{sp}\left\{\left(\begin{array}{c}
1 \\
-1 \\
1 \\
-1
\end{array}\right)\right\}, \quad \mu_{2} \in \operatorname{sp}\left\{\left(\begin{array}{c}
1 \\
1 \\
-1 \\
-1
\end{array}\right)\right\}, \\
& \mu_{3} \in \operatorname{sp}\left\{\left(\begin{array}{c}
1 \\
-1 \\
-1 \\
1
\end{array}\right)\right\}, \quad \mu_{4} \in \operatorname{sp}\left\{\left(\begin{array}{l}
1 \\
1 \\
1 \\
1
\end{array}\right)\right\} .
\end{aligned}
$$

The eigenvalues of $I-A$ are obviously $1-\lambda_{i}$ with right eigenvectors $\mu_{i}$. Theorems 5 and 6 imply that each set $\operatorname{ker}\left(I_{4 n}-\Pi_{j} \otimes I_{n}\right), j=1,2,3$ contains a globally asymptotically stable subset. With the use of Corollary 8 (or Corollary 9), we can show that the conditions for stability of these partial synchronization manifolds do not completely coincide with the conditions for which we can guarantee full synchronization.

First, to have $\sigma^{*}<\bar{\sigma}$ (or $\sigma^{\prime}<\bar{\sigma}$ ) requires the eigenvectors $\mu_{1}$ and $\mu_{2}$ to be in range $\left(I-\Pi_{j}\right)$ while eigenvector $\mu_{3}$ is not in range $\left(I-\Pi_{j}\right)$. (Note that $1-\lambda_{3}$ is indeed the smallest nonzero eigenvalue of $L=I-A$.) This only holds true for $\Pi_{3}$. Thus, we can find values for the coupling strength for which systems 1 and 4 and systems 2 and 3 definitely synchronize, and these values for the coupling strength are smaller than the value of the coupling strength that guarantees full synchronization. In other words, for values of the coupling strength $\sigma$ such that $\sigma^{*}<\sigma \leq \bar{\sigma}$ (or $\sigma^{\prime}<\sigma \leq \bar{\sigma}$ ), we guarantee that systems 1 and 4 and systems 2 and 3 synchronize but it is not necessarily true that this also implies full synchronization. Hence, this partial synchronization mode may be observed.

Second, to have $\gamma^{*}>\bar{\gamma}$ (or $\gamma^{\prime}>\bar{\gamma}$ ), we need the eigenvectors $\mu_{2}$ and $\mu_{3}$ to be in range $\left(I-\Pi_{j}\right)$ while eigenvector $\mu_{1}$ is not in range $\left(I-\Pi_{j}\right)$. This only holds true for $\Pi_{2}$. This implies that synchronization of systems 1 and 3 and systems 2 and 4 can be guaranteed for values of the product of the coupling strength and time-delay larger than the one which guarantees full synchronization, i.e., given a fixed $\sigma$ being sufficiently large, synchronization of systems 1 and 3 and systems 2 and 4 is guaranteed for time-delays larger than those for which full synchronization is guaranteed. Thus, the conditions for stability of this partial synchronization mode do not necessarily coincide with the conditions for full synchronization, which implies that this partial synchronization mode may indeed be observed.

Figure 4 shows the results of numerical simulations of the network with Hindmarsh-Rose neurons (14) that interact via coupling I (8) for different values of $\sigma$ and $\tau$. The left panels show the output trajectories for the last 500 time units. Note that $y_{i}(t)=y_{j}(t)$ implies $z_{i}(t)=z_{j}(t)$ since the in- ternal dynamics are convergent. Thus, it is sufficient to show only the output trajectories. The other figures show these output trajectories for the last 500 time units in the $y_{i}, y_{j}$-plane. We have $y_{i}(t)=y_{j}(t)$ only if the graph is (a subset of) the diagonal. Figure 4(a) shows results for $\sigma=1$ and $\tau=0.5$. It can be seen that neuron 1 synchronizes with neuron 4 and at the same time will neuron 2 synchronize with neuron 3 . Figure 4(b) shows that all neurons are synchronized if the coupling strength is increased to $\sigma=3$ while $\tau$ remains the same $(\tau=0.5)$. For $\sigma=3$ and $\tau=2$, neurons 1 and 3 synchronize and neurons 2 and 4 synchronize. See, Figure 4(c). These numerical results are explained by analysis presented above.

\section{B. Network of example 2}

In this example, we assume that the neurons interact via nondelayed coupling, i.e., coupling (8) or (9) with $\tau=0$.
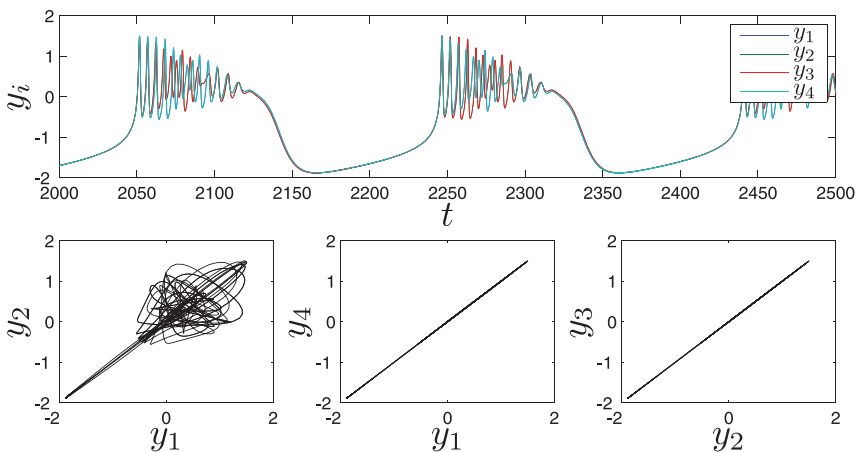

(a)
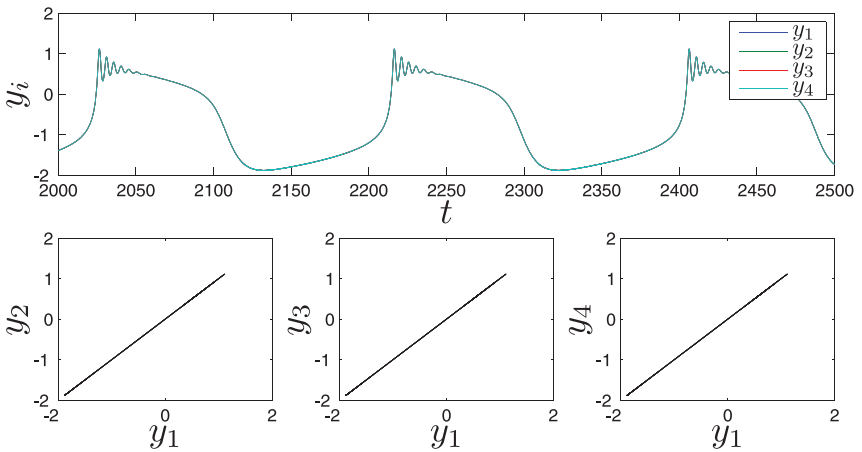

(b)
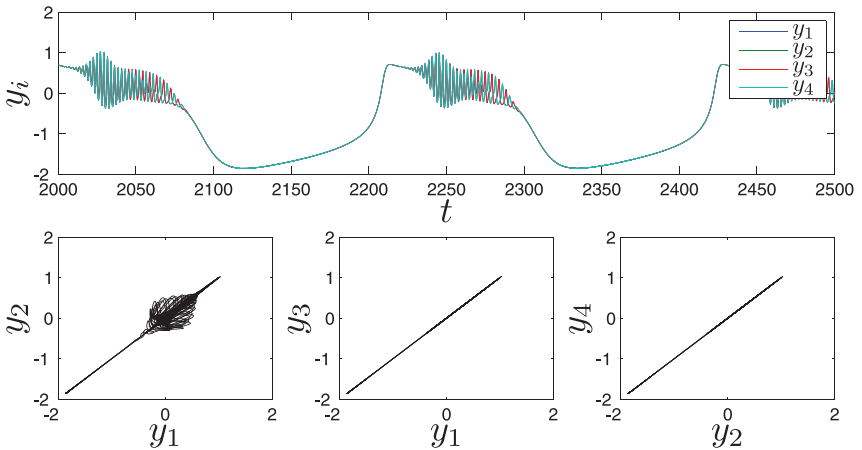

(c)

FIG. 4. Partial and full synchronization in a network with four HindmarshRose neurons. (a) Partial synchronization of neurons 1 and 4 and neurons 2 and 3 for $\sigma=1, \tau=0.5$. (b) Full synchronization for $\sigma=3, \tau=0.5$. (c) Partial synchronization of neurons 1 and 3 and neurons 2 and 4 for $\sigma=3, \tau=2$. 
Consider the network of example 2 with $a_{1}=a_{3}=\frac{4}{11}$ and $a_{2}=\frac{1}{11} a_{4}=\frac{2}{11}$. The Laplacian matrix is

$$
L=\frac{1}{11}\left(\begin{array}{cccccccc}
11 & -4 & 0 & -4 & -1 & -2 & 0 & 0 \\
-4 & 11 & -4 & 0 & -2 & -1 & 0 & 0 \\
0 & -4 & 11 & -4 & 0 & 0 & -1 & -2 \\
-4 & 0 & -4 & 11 & 0 & 0 & -2 & -1 \\
-1 & -2 & 0 & 0 & 11 & -4 & 0 & -4 \\
-2 & -1 & 0 & 0 & -4 & 11 & -4 & 0 \\
0 & 0 & -1 & -2 & 0 & -4 & 11 & -4 \\
0 & 0 & -2 & -1 & -4 & 0 & -4 & 11
\end{array}\right) .
$$

The permutation matrix

$$
\Pi=\left(\begin{array}{llllllll}
0 & 1 & 0 & 0 & 0 & 0 & 0 & 0 \\
0 & 0 & 1 & 0 & 0 & 0 & 0 & 0 \\
0 & 0 & 0 & 1 & 0 & 0 & 0 & 0 \\
1 & 0 & 0 & 0 & 0 & 0 & 0 & 0 \\
0 & 0 & 0 & 0 & 0 & 1 & 0 & 0 \\
0 & 0 & 0 & 0 & 0 & 0 & 1 & 0 \\
0 & 0 & 0 & 0 & 0 & 0 & 0 & 1 \\
0 & 0 & 0 & 0 & 1 & 0 & 0 & 0
\end{array}\right)
$$

does not commute with $L=D-A$ but there exists a solution $X$ of the matrix equation $(I-\Pi) L=X(I-\Pi)$. Thus, the set $\operatorname{ker}\left(I_{8 n}-\Pi \otimes I_{n}\right)$ defines a linear invariant manifold for the coupled systems (7) and (9). Using a pseudo inverse of $I-\Pi$, we compute

$$
X=\frac{1}{44}\left(\begin{array}{rrrrrrrr}
41 & -19 & -3 & -19 & 3 & -1 & -1 & -1 \\
-19 & 41 & -19 & -3 & -1 & -5 & -1 & 7 \\
-3 & -19 & 41 & -19 & -1 & -1 & 3 & -1 \\
-19 & -3 & -19 & 41 & -1 & 7 & -1 & -5 \\
3 & -1 & -1 & -1 & 41 & -19 & -3 & -19 \\
-1 & -5 & -1 & 7 & -19 & 41 & -19 & -3 \\
-1 & -1 & 3 & -1 & -3 & -19 & 41 & -19 \\
-1 & 7 & -10 & -5 & -19 & -3 & -19 & 41
\end{array}\right)
$$

Consider the singular value decomposition of $I-\Pi$, cf. Ref. $61, I-\Pi=U \Sigma V^{\top}$ with unitary matrices $U$ and $V$, and $\Sigma$ is a diagonal matrix with the singular vales of $I-\Pi$ as entries. Note that dim $\operatorname{ker}(I-\Pi)=2$. Assume that $\Sigma$ has the form

$$
\Sigma=\left(\begin{array}{cc}
\Sigma_{1} & 0 \\
0 & 0
\end{array}\right)
$$

with $\Sigma_{1} \in \mathbb{R}^{6 \times 6}$ diagonal matrix with the nonzero singular values of $I-\Pi$ as entries. Let,

$$
U^{\top} X U=\left(\begin{array}{cc}
X_{1} & X_{2}^{\top} \\
X_{2} & X_{3}
\end{array}\right)
$$

then, for every $\vartheta \in \mathbb{R}^{k}$,

$$
\vartheta^{\top}(I-\Pi)^{\top} X(I-\Pi) \vartheta \geq c|(I-\Pi) \vartheta|^{2},
$$

with $c=\frac{32}{44}$ being the smallest eigenvalue of the matrix $X_{1}$. Since the smallest nonzero eigenvalue of $L$ equals $\frac{24}{44}$, we know that our condition for stability of the partial synchronization manifold does not coincide with stability of the full synchronization manifold. The results of numerical simulations, which are explained by our analysis, are shown in Figure 5.

\section{A hierarchical network}

The last example is a network in which a hierarchical structure is present. Consider the network shown in Figure 6.
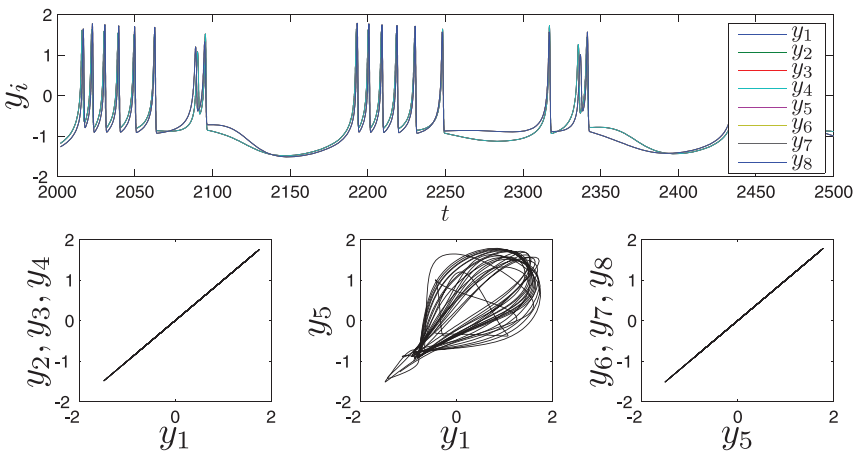

(a)
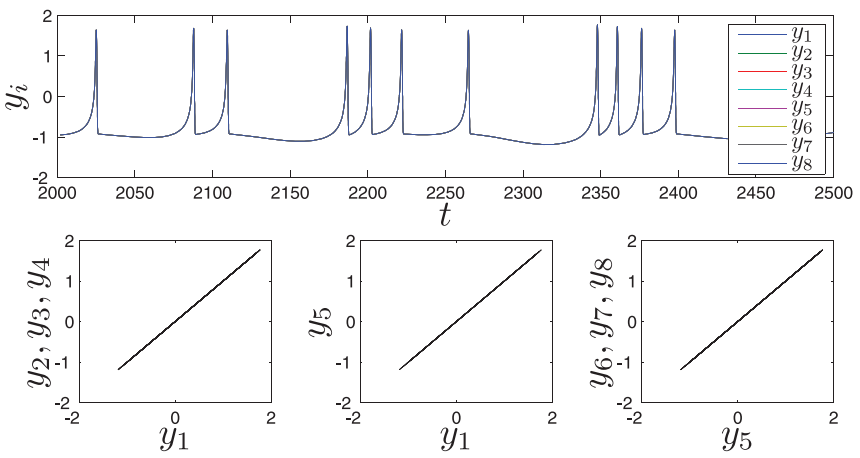

(b)

G. 5. Partial and full synchronization of Hindmarsh-Rose neurons in the network of example 2. (a) Partial synchronization of neurons 1, 2, 3, and 4 for $\sigma=2($ and $\tau=0)$.

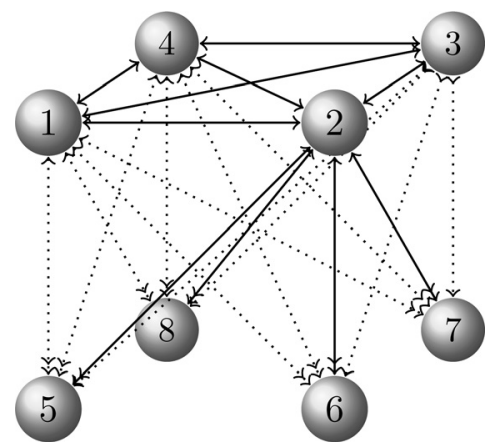

FIG. 6. A hierarchical network. Each node in the top layer couples (nodes 1, 2,3 , and 4) to every other node in the top layer with weight $\frac{1}{20}$ and to each node in the bottom layer (nodes 5, 6, 7, and 8) with weight $\frac{1}{4}$. Each node in the bottom layer only couples to each node in the top layer with weight $\frac{1}{20}$. This asymmetric coupling between nodes in the top layer and bottom layer is indicated by $\rightarrow \leftrightarrow$. There is no difference between the solid and dotted lines; the latter are used merely for clarity of presentation. 
The four neurons in the top layer, neurons $1,2,3$ and 4, connect to every other node in the top layer with interconnection weight $\frac{1}{20}$. These neurons also connect to every neuron in the bottom layer, i.e., neurons $5,6,7$, and 8 , with interconnection weight $\frac{1}{4}$. The neurons in the bottom layer only connect to the neurons in the top layer. The weights of these connections are all $\frac{1}{20}$. The corresponding Laplacian matrix is

$$
L=\frac{1}{20}\left(\begin{array}{cccccccc}
7 & -1 & -1 & -1 & -1 & -1 & -1 & -1 \\
-1 & 7 & -1 & -1 & -1 & -1 & -1 & -1 \\
-1 & -1 & 7 & -1 & -1 & -1 & -1 & -1 \\
-1 & -1 & -1 & 7 & -1 & -1 & -1 & -1 \\
-5 & -5 & -5 & -5 & 20 & 0 & 0 & 0 \\
-5 & -5 & -5 & -5 & 0 & 20 & 0 & 0 \\
-5 & -5 & -5 & -5 & 0 & 0 & 20 & 0 \\
-5 & -5 & -5 & -5 & 0 & 0 & 0 & 20
\end{array}\right)
$$

We can only give statements for systems that interact via coupling II since the diagonal entries of $L$ are not all identical.

Let $\lambda_{i}$ be an eigenvalue of $L$ with eigenvector $\mu_{i}$. A straightforward computation shows that $L$ has eigenvalues

$$
\lambda_{1}=0, \quad \lambda_{2}=\frac{2}{5}, \quad \lambda_{3}=\frac{2}{5}, \quad \lambda_{4}=\frac{2}{5}, \quad \lambda_{5}=1, \quad \lambda_{6}=1, \quad \lambda_{7}=1, \quad \lambda_{8}=\frac{6}{5},
$$

with eigenvectors

$$
\begin{aligned}
& \left.\left.\mu_{1} \in \operatorname{sp}\left\{\left(\begin{array}{l}
1 \\
1 \\
1 \\
1 \\
1 \\
1 \\
1 \\
1
\end{array}\right)\right\}, \quad \mu_{2} \in \operatorname{sp}\left\{\left(\begin{array}{c}
1 \\
-1 \\
0 \\
0 \\
0 \\
0 \\
0 \\
0
\end{array}\right)\right\}, \quad \mu_{3} \in \operatorname{sp}\left\{\begin{array}{c}
1 \\
0 \\
-1 \\
0 \\
0 \\
0 \\
0 \\
0
\end{array}\right)\right\}, \quad \mu_{4} \in \operatorname{sp}\left\{\begin{array}{c}
1 \\
0 \\
0 \\
-1 \\
0 \\
0 \\
0 \\
0
\end{array}\right)\right\}, \\
& \left.\mu_{5} \in \mathrm{sp}\left\{\left(\begin{array}{c}
0 \\
0 \\
0 \\
0 \\
1 \\
-1 \\
0 \\
0
\end{array}\right)\right\}, \quad \mu_{6} \in \mathrm{sp}\left\{\left(\begin{array}{c}
0 \\
0 \\
0 \\
0 \\
1 \\
0 \\
-1 \\
0
\end{array}\right)\right\}, \quad \mu_{7} \in \mathrm{sp}\left\{\left(\begin{array}{c}
0 \\
0 \\
0 \\
0 \\
1 \\
0 \\
0 \\
-1
\end{array}\right)\right\}, \quad \mu_{8} \in \mathrm{sp}\left\{\begin{array}{c}
1 \\
1 \\
1 \\
1 \\
-5 \\
-5 \\
-5 \\
-5
\end{array}\right)\right\} .
\end{aligned}
$$

The network contains many symmetries but the only relevant symmetries for partial synchronization are given by the permutation matrices

$$
\Pi_{1}=\left(\begin{array}{cc}
I & \mathbf{0} \\
\mathbf{0} & R
\end{array}\right) \text { and } \Pi_{2}=\left(\begin{array}{cc}
\mathrm{R} & \mathbf{0} \\
\mathbf{0} & \mathrm{R}
\end{array}\right),
$$

with

$$
\mathbf{0}=\left(\begin{array}{llll}
0 & 0 & 0 & 0 \\
0 & 0 & 0 & 0 \\
0 & 0 & 0 & 0 \\
0 & 0 & 0 & 0
\end{array}\right) \text { and } \mathrm{R}=\left(\begin{array}{cccc}
0 & 1 & 0 & 0 \\
0 & 0 & 1 & 0 \\
0 & 0 & 0 & 1 \\
1 & 0 & 0 & 0
\end{array}\right)
$$

Other permutation matrices will either define the same linear invariant manifolds and/or the conditions for attractivity of these manifolds will coincide with the conditions for other 

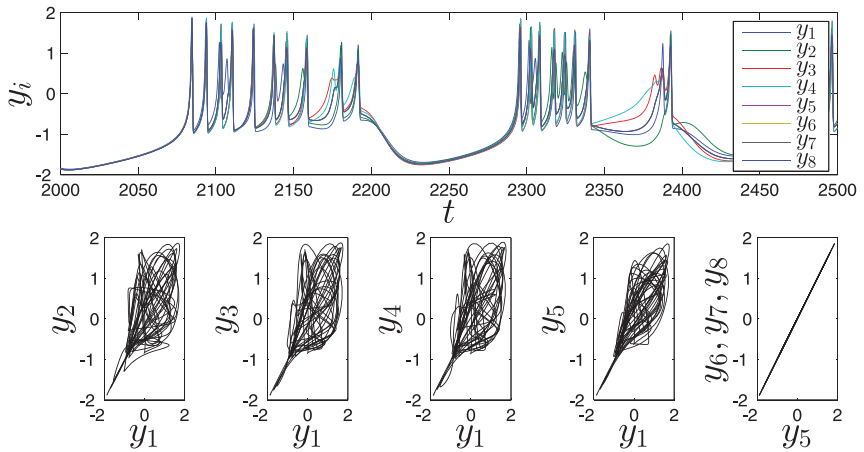

(a)
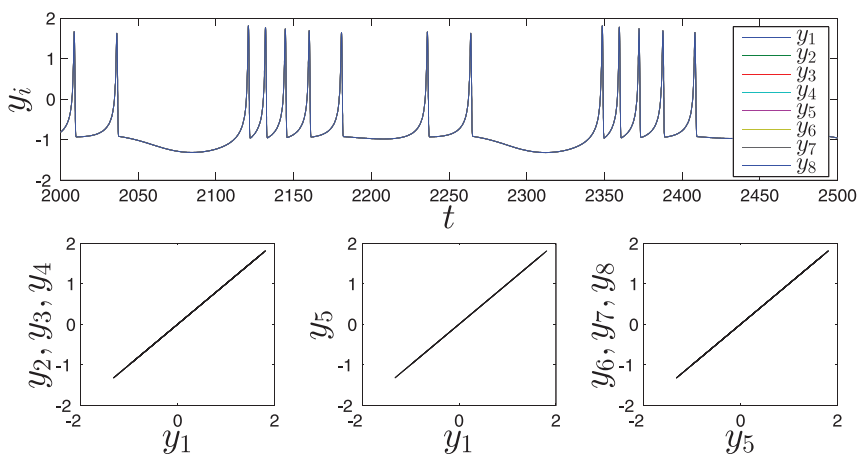

(b)
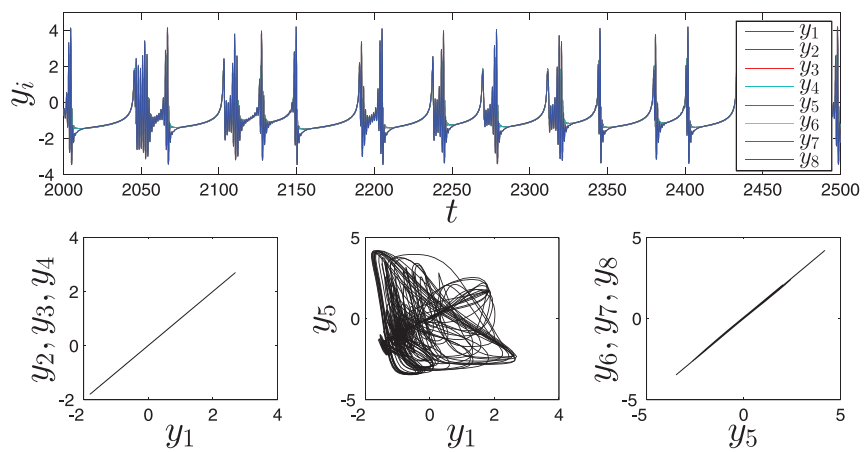

(c)

FIG. 7. Partial and full synchronization in the network shown in Figure 6. (a) Partial synchronization of neurons $5,6,7$, and 8 for $\sigma=2, \tau=0.1$. There is no synchronization of any other neurons. (b) Full synchronization for $\sigma=8, \tau=0.1$. (c) Partial synchronization of neurons $1,2,3$, and 4 and neurons 5, 6, 7, and 8 for $\sigma=8, \tau=0.5$.

manifolds to be attracting. It is easy to see that $\mu_{2}, \mu_{3}, \mu_{4} \in$ $\operatorname{range}\left(I-\Pi_{2}\right)$ and $\mu_{5}, \mu_{6}, \mu_{7} \in \operatorname{range}\left(I-\Pi_{1}\right)$. Since $\lambda_{2}=$ $\lambda_{3}=\lambda_{4}=\frac{2}{5}$ and $\lambda_{5}=\lambda_{6}=\lambda_{7}$ are all larger than $\lambda_{2}$ but smaller than $\lambda_{8}$, we can have partial synchronization. The numerical simulations with the Hindmarsh-Rose neurons shown in Figure 7 support our analysis.

\section{CONCLUSIONS AND DISCUSSION}

We have presented a framework for analyzing the emergence of partial synchronization in oscillator networks of which the units interact via time-delay diffusive coupling. It is shown that symmetries present in the network define linear invariant manifold which, when being attracting, define modes of partial synchronization. For both types of diffusive time-delay coupling, i.e., coupling I and coupling II, condi- tions are presented that guarantee such linear invariant manifolds to be globally stable. Global synchronization of the whole network can be defined as the union of all partial synchronization modes, hence all units of the network synchronize if all partial synchronization modes are stable. For a particular class of networks, we have presented necessary conditions for the linear invariant manifold to be stable without having stability of the full synchronization manifold. Numerical simulations with networks of diffusively time-delay coupled Hindmarsh-Rose neurons demonstrate our results.

In this paper, we have considered diffusive coupling subject to a single time-delay. The most straightforward extension of our results would be to include multiple time-delays. It is not hard to imagine that symmetries with respect to time-delays can, in combination with symmetries in interaction weights, contribute to the emergence of partial synchronization modes. The following theorem presents a simple result in this direction.

Theorem 10. Let $\mathbb{P}_{k}$ be the set of commuting symmetric $k \times k$ dimensional permutation matrices with zero trace. (Note that the zero trace assumption implies $k$ is an even integer.) Consider $k$ coupled systems (7), which interact via coupling functions

$$
u(t)=-\sigma y(t)+\sigma \sum_{\ell} \eta_{\ell} \Pi_{\ell} y\left(t-\tau_{\ell}\right)
$$

with $\Pi_{\ell} \in \mathbb{P}_{k}, \eta_{\ell}>0$ and $\sum_{\ell} \eta_{\ell}=1$. Assume that $A=\sum_{\ell} \eta_{\ell} \Pi_{\ell}$ is irreducible. Then for each $\Pi_{\ell}$, the set $\operatorname{ker}\left(I_{k n}-\Pi_{\ell} \otimes I_{n}\right)$ defines a linear invariant manifold for the coupled systems. Suppose in addition that

- the systems (7) are strictly semipassive with a radially unbounded storage function;

- the systems have the normal form (13) and there is a symmetric matrix $P \in \mathbb{R}^{k \times k}$, such that the eigenvalues of the symmetric matrix

$$
\left[\frac{\partial q}{\partial z_{i}}\left(z_{i}, y_{i}\right)\right]^{\top} P+P\left[\frac{\partial q}{\partial z_{i}}\left(z_{i}, y_{i}\right)\right]
$$

are negative and bounded away from zero for all $z_{i} \in \mathbb{R}^{p}$ and $y_{i} \in \mathbb{R}^{m}$.

Then, the solutions of the coupled systems are ultimately bounded and there exist positive constants $\sigma_{\ell}^{\diamond}$ and $\gamma_{\ell}^{\diamond}$, such that if $\sigma>\sigma_{\ell}^{\diamond}$ and $\sigma \tau_{\ell}<\gamma_{\ell}$, then the set $\operatorname{ker}\left(I_{k n}-\Pi_{\ell} \otimes I_{n}\right)$ contains a globally attractive subset.

The proof is presented in the Appendix.

Example 4. Consider $k=4$, coupled systems in a ring with coupling functions

$u(t)=-\sigma y(t)+\frac{\sigma}{2}\left(\Pi_{1} \otimes I_{n}\right) y\left(t-\tau_{1}\right)+\frac{\sigma}{2}\left(\Pi_{2} \otimes I_{n}\right) y\left(t-\tau_{2}\right)$

with

$$
\Pi_{1}=\left(\begin{array}{llll}
0 & 1 & 0 & 0 \\
1 & 0 & 0 & 0 \\
0 & 0 & 0 & 1 \\
0 & 0 & 1 & 0
\end{array}\right) \text { and } \Pi_{2}=\left(\begin{array}{llll}
0 & 0 & 0 & 1 \\
0 & 0 & 1 & 0 \\
0 & 1 & 0 & 0 \\
1 & 0 & 0 & 0
\end{array}\right)
$$

Applying Theorem 10, we conclude that if the systems are strictly semipassive and have convergent internal dynamics, 


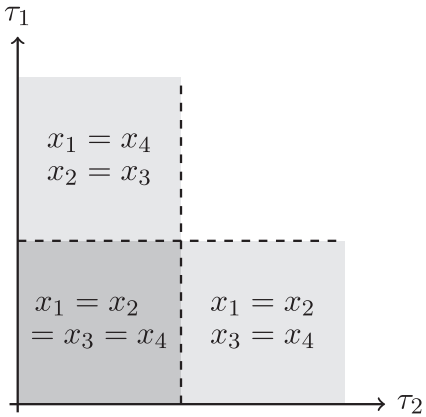

FIG. 8. Partial synchronization in a network with different time-delays for some sufficiently large $\sigma$. Systems 1 and 2 and systems 3 and 4 synchronize for any $\tau_{2} \geq 0$ if $\tau_{1}$ is sufficiently small. Obviously, systems 1 and 4 and systems 2 and 3 synchronize for any $\tau_{1} \geq 0$ if $\tau_{2}$ is sufficiently small. Full synchronization is achieved if both $\tau_{1}$ and $\tau_{2}$ are sufficiently small.

then systems 1 and 2 synchronize and systems 3 and 4 synchronize for sufficiently large $\sigma$ and $\sigma \tau_{1}$ sufficiently small. This result is independent of the value of $\tau_{2}$. Due to the symmetry, we also have that systems 1 and 4 synchronize and systems 2 and 3 synchronize for sufficiently large $\sigma$ and $\sigma \tau_{2}$ sufficiently small for every $\tau_{1} \geq 0$. If $\sigma$ is sufficiently large and both $\sigma \tau_{1}$ and $\sigma \tau_{2}$ are sufficiently small, full synchronization is guaranteed. Figure 8 summarizes these results for fixed sufficiently large $\sigma$.

Another possible extension is to allow for time-varying interaction weights and/or time-varying delays. From a practical point of view, it might be interesting to introduce weaker notions of partial synchronization than the one used in this paper. In practice, it is often sufficient that the units in a cluster behave similarly enough, e.g.,

$$
\limsup _{t \rightarrow \infty}\left|x_{i}(t)-x_{j}(t)\right|<\varepsilon,
$$

for at least one pair $(i, j)$ for some sufficiently small constant $\varepsilon>0$. (How small $\varepsilon$ should be depends of course on the application.) Using a weaker notion of (partial) synchronization enables the development of similar theories for heterogeneous populations, which should be of great practical interest. Using a weaker notion of partial synchronization would also allow for less restrictive assumptions on the network, e.g., weak partial synchronization might emerge in networks without perfect symmetries. Recent developments on the identification of clustering in networks, cf. Refs. 62 and 63, might then be used to establish new results for practical partial synchronization.

\section{ACKNOWLEDGMENTS}

C.v.L. was supported by an Odysseus grant from the Research Foundation Flanders (FWO).

\section{APPENDIX A: PROOFS OF THEOREM 5, THEOREM 6, AND THEOREM 10}

Proof of Theorem 5. Note that $z(t) \in \operatorname{ker}\left(I_{k p}-\Pi \otimes I_{p}\right)$ and $y(t) \in \operatorname{ker}\left(I_{k m}-\Pi \otimes I_{m}\right)$ define equations

$$
\begin{aligned}
& z_{i}(t)-z_{j}(t)=0, \\
& y_{i}(t)-y_{j}(t)=0,
\end{aligned}
$$

for pairs $(i, j)$ with $i, j \in\{1,2, \ldots, k\}$. Let $\mathcal{I}_{\Pi}$ be such that Eq. (A1) holds for every $(i, j) \in \mathcal{I}_{\Pi}$. Let $\xi(t)$ be the vector with as entries the constraint Eq. (A1a) and, similarly, the constraint Eq. (A1b) are stored in the vector $\eta(t)$. We want to show that $\xi \equiv 0$ and $\eta \equiv 0$ are globally asymptotically stable under the conditions supplied in the theorem. We will do so by constructing a positive definite Lyapunov-Razumikhin function of the form

$$
V(\xi(t), \eta(t))=V_{1}(\xi(t))+V_{2}(\eta(t))
$$

and showing that our assumptions imply that

$$
\begin{aligned}
\dot{V}(\xi(t), \eta(t))= & \limsup _{h \rightarrow 0_{+}} \frac{1}{h}[V(\xi(t+h), \eta(t+h)) \\
& -V(\xi(t), \eta(t))]<0
\end{aligned}
$$

whenever

$$
w(V(\xi(t), \eta(t)))>V(\xi(t+\theta), \eta(t+\theta)), \quad \theta \in[-\tau, 0],
$$

with $w: \mathbb{R}_{>0} \rightarrow \mathbb{R}_{>0}, w(s)>s$ and $w(0)=0$. See, for instance, Ref. 64 for details about Lyapunov-Razumikhin functions. Let

$$
\begin{aligned}
V_{1}(\xi(t)) & =\xi^{\top}(t)(I \otimes P) \xi(t) \\
& =\sum_{(i, j) \in \mathcal{I}_{\Pi}}\left(z_{i}(t)-z_{j}(t)\right)^{\top} P\left(z_{i}(t)-z_{j}(t)\right) .
\end{aligned}
$$

Then,

$$
\begin{aligned}
\dot{V}_{1}(\xi(t))= & \sum_{(i, j) \in \mathcal{I}_{\Pi}}\left(z_{i}(t)-z_{j}(t)\right)^{\top} P\left[q\left(z_{i}(t), y_{i}(t)\right)\right. \\
& \left.-q\left(z_{j}(t), y_{j}(t)\right)\right]+\left[q\left(z_{i}(t), y_{i}(t)\right)\right. \\
& \left.-q\left(z_{j}(t), y_{j}(t)\right)\right]^{\top} P\left(z_{i}(t)-z_{j}(t)\right) .
\end{aligned}
$$

We can write

$$
\begin{aligned}
& \left(z_{i}(t)-z_{j}(t)\right)^{\top} P\left(q\left(z_{i}(t), y_{i}(t)\right)-q\left(z_{j}(t), y_{j}(t)\right)\right) \\
& \quad=\left(z_{i}(t)-z_{j}(t)\right)^{\top} P\left(q\left(z_{i}(t), y_{i}(t)\right)-q\left(z_{j}(t), y_{i}(t)\right)\right) \\
& \quad+\left(z_{i}(t)-z_{j}(t)\right)^{\top} P\left(q\left(z_{j}(t), y_{i}(t)\right)-q\left(z_{j}(t), y_{j}(t)\right)\right) .
\end{aligned}
$$

Denote

$$
\Phi_{1}\left(\zeta_{1}\right)=\left(z_{i}(t)-z_{j}(t)\right)^{\top} P q\left(z_{j}(t)+\zeta_{1}\left(z_{i}(t)-z_{j}(t)\right), y_{i}(t)\right),
$$

$$
\Phi_{2}\left(\zeta_{2}\right)=\left(z_{i}(t)-z_{j}(t)\right)^{\top} P q\left(z_{j}(t), y_{j}(t)+\zeta_{2}\left(y_{i}(t)-y_{j}(t)\right)\right),
$$

with $\zeta_{1} \in[0,1], \zeta_{2} \in[0,1]$ and note that Eq. (A7) can also be written as $\Phi_{1}(1)-\Phi_{1}(0)+\Phi_{2}(1)-\Phi_{2}(0)$. Invoking the mean value theorem and using the fact that $q$ is sufficiently smooth, we have

$$
\begin{aligned}
\Phi_{1}(1)-\Phi_{1}(0) & =\frac{\partial \Phi_{1}}{\partial \zeta_{1}}\left(\zeta_{1}^{*}\right), & \zeta_{1}^{*} \in[0,1], \\
\Phi_{2}(1)-\Phi_{2}(0) & =\frac{\partial \Phi_{2}}{\partial \zeta_{2}}\left(\zeta_{2}^{*}\right), & \zeta_{2}^{*} \in[0,1],
\end{aligned}
$$




$$
\begin{aligned}
\frac{\partial \Phi_{1}}{\partial \zeta_{1}}\left(\zeta_{1}^{*}\right)= & \left(z_{i}(t)-z_{j}(t)\right)^{\top} P \\
& \times\left[\frac{\partial q}{\partial z}\left(z_{j}(t)+\zeta_{1}^{*}\left(z_{i}(t)-z_{j}(t)\right), y_{i}(t)\right)\right] \\
& \times\left(z_{i}(t)-z_{j}(t)\right), \\
\frac{\partial \Phi_{2}}{\partial \zeta_{2}}\left(\zeta_{2}^{*}\right)= & \left(z_{i}(t)-z_{j}(t)\right)^{\top} P \\
& \times\left[\frac{\partial q}{\partial y}\left(z_{j}(t), y_{j}(t)+\zeta_{2}^{*}\left(y_{i}(t)-y_{j}(t)\right)\right)\right] \\
& \times\left(y_{i}(t)-y_{j}(t)\right) .
\end{aligned}
$$

Invoking boundedness of all solutions (by Lemma 2), hence the matrix $\frac{\partial q}{\partial y}(\cdot, \cdot)$ is bounded, and using the assumption that

$$
\frac{\partial q^{\top}}{\partial z}(\cdot, \cdot) P+P \frac{\partial q}{\partial z}(\cdot, \cdot)
$$

is uniformly negative definite, there will exist positive constants $c_{1}, c_{2}$ such that

$$
\dot{V}_{1}(\xi(t)) \leq-c_{1}|\xi(t)|^{2}+c_{2}|\xi(t)||\eta(t)| .
$$

Let

$$
\begin{aligned}
V_{2}(\eta(t)) & =\frac{1}{2} \eta^{\top}(t) \eta(t) \\
& =\frac{1}{2} \sum_{(i, j) \in \mathcal{I}_{\Pi}}\left(y_{i}(t)-y_{j}(t)\right)^{\top}\left(y_{i}(t)-y_{j}(t)\right) \\
& =\frac{1}{2}\left[\left(I_{k m}-\Pi \otimes I_{m}\right) y(t)\right]^{\top}\left[\left(I_{k m}-\Pi \otimes I_{m}\right) y(t)\right] .
\end{aligned}
$$

Hence

$\dot{V}_{2}(\eta(t))=\eta^{\top}(t) \dot{\eta}(t)=\left[\left(I_{k m}-\Pi \otimes I_{m}\right) y(t)\right]^{\top}\left[\left(I_{k m}-\Pi \otimes I_{m}\right) \dot{y}(t)\right]$.

Using the assumption that there is a $X$ such that $\left(I_{k}-\Pi\right) A$ $=X\left(I_{k}-\Pi\right)$ (by Lemma 3), we can write

$$
\begin{aligned}
\left(I_{k m}-\Pi \otimes I_{m}\right) \dot{y}(t)= & \left(I_{k m}-\Pi \otimes I_{m}\right)[a(y(t), z(t))-\sigma y(t)] \\
& +\sigma\left(I_{k m}-\Pi \otimes I_{m}\right)\left(A \otimes I_{m}\right) y(t-\tau) \\
= & \left(I_{k m}-\Pi \otimes I_{m}\right) a(y(t), z(t)) \\
& -\sigma\left(I_{k m}-\Pi \otimes I_{m}\right) y(t) \\
& +\sigma\left(X \otimes I_{m}\right)\left(I_{k m}-\Pi \otimes I_{m}\right) y(t-\tau),
\end{aligned}
$$

with $\quad a(y(t), z(t))=\operatorname{col}\left(a\left(y_{1}(t), z_{1}(t)\right), \ldots, a\left(y_{k}(t), z_{k}(t)\right)\right)$. (Note the abuse of notation, however, no confusion should arise.) Using Leibniz's rule, we can write

$$
y(t-\tau)=y(t)-\int_{-\tau}^{0} \dot{y}(t+s) \mathrm{d} s,
$$

and substitution of Eq. (A17) in the right hand side of Eq. (A16) yields

$$
\begin{aligned}
& \left(I_{k m}-\Pi \otimes I_{m}\right) a(y(t), z(t))-\sigma\left(I_{k m}-X \otimes I_{m}\right)\left(I_{k m}-\Pi \otimes I_{m}\right) y(t) \\
& -\sigma\left(X \otimes I_{m}\right)\left(I_{k m}-\Pi \otimes I_{m}\right) \int_{-\tau}^{0} \dot{y}(t+s) \mathrm{d} s .
\end{aligned}
$$

It follows that the right hand side of Eq. (A15) is the sum of the three terms

$$
\left[\left(I_{k m}-\Pi \otimes I_{m}\right) y(t)\right]^{\top}\left[\left(I_{k m}-\Pi \otimes I_{m}\right) a(y(t), z(t))\right],
$$

$$
-\sigma\left[\left(I_{k m}-\Pi \otimes I_{m}\right) y(t)\right]^{\top}\left(I_{k m}-X \otimes I_{m}\right)\left[\left(I_{k m}-\Pi \otimes I_{m}\right) y(t)\right],
$$

$$
\begin{aligned}
& -\sigma \int_{-\tau}^{0}\left[\left(I_{k m}-\Pi \otimes I_{m}\right) y(t)\right]^{\top}\left(X \otimes I_{m}\right) \\
& \quad \times\left[\left(I_{k m}-\Pi \otimes I_{m}\right) \dot{y}(t+s) \mathrm{d} s\right] .
\end{aligned}
$$

First, using the boundedness of solutions (Lemma 2) and the function $a$ being sufficiently smooth, one concludes that there exist positive constants $c_{3}, c_{4}$ such that

$$
\begin{aligned}
& {\left[\left(I_{k m}-\Pi \otimes I_{m}\right) y(t)\right]^{\top}\left[\left(I_{k m}-\Pi \otimes I_{m}\right) a(y(t), z(t))\right]} \\
& \quad \leq c_{3}|\eta(t)|^{2}+c_{4}|\eta(t)||\xi(t)| .
\end{aligned}
$$

(See the machinery preceding Eq. (A13).) Second, by assumption there is a constant $c^{*}>0$ such that

$$
\begin{aligned}
& -\sigma\left[\left(I_{k m}-\Pi \otimes I_{m}\right) y(t)\right]^{\top}\left(I_{k m}-X \otimes I_{m}\right)\left[\left(I_{k m}-\Pi \otimes I_{m}\right) y(t)\right] \\
& \quad \leq-\sigma c^{*}|\eta(t)|^{2} .
\end{aligned}
$$

Third, letting $w(s)=\kappa^{2} s^{2}$ with constant $\kappa>1$, we have

$$
\begin{aligned}
- & \sigma \int_{-\tau}^{0}\left[\left(I_{k m}-\Pi \otimes I_{m}\right) y(t)\right]^{\top}\left(X \otimes I_{m}\right)\left[\left(I_{k m}-\Pi \otimes I_{m}\right) \dot{y}(t+s) \mathrm{d} s\right]-\sigma \int_{-\tau}^{0}\left[\left(I_{k m}-\Pi \otimes I_{m}\right) y(t)\right]^{\top}\left(X \otimes I_{m}\right)\left[\left(I_{k m}-\Pi \otimes I_{m}\right)\right. \\
& \times[a(y(t+s), z(t+s))-\sigma y(t+s)+\sigma(A \otimes I) y(t+s-\tau)] \mathrm{d} s] \\
& \leq \kappa \sigma \tau\|X\|\left(c_{3}|\eta(t)|^{2}+c_{4}|\eta(t)||\xi(t)|+\sigma|\eta(t)|^{2}+\sigma \frac{\left\|X^{2}\right\|}{\|X\|}|\eta(t)|^{2}\right) .
\end{aligned}
$$


To get the latter inequality, we have used $\left(I_{k}-\Pi\right) A=X$ $\left(I_{k}-\Pi\right)$ and

$$
\begin{aligned}
& w(V(\xi(t), \eta(t)))>V(\xi(t+\theta), \eta(t+\theta)) \\
& \quad \Rightarrow \kappa|\eta(t)| \geq|\eta(t+\theta)| .
\end{aligned}
$$

Denote $\gamma=\sigma \tau$ such that, combining Eqs. (A22), (A23), and (A24),

$$
\begin{aligned}
& \dot{V}_{2}(\eta) \leq\left(c_{3}(1+\kappa \gamma|| X||)+\sigma \kappa \gamma\left(\|X\|+\left\|X^{2}\right\|\right)-\sigma c^{*}\right)|\eta(t)|^{2} \\
& \quad+c_{4}(1+\kappa \gamma|| X||)|\eta(t)||\xi(t)|
\end{aligned}
$$

if $\kappa^{2} V(\xi(t), \eta(t))>V(\xi(t+\theta), \eta(t+\theta)), \theta \in[-2 \tau, 0]$. From Eqs. (A13) and (A26), we conclude that, if $\kappa^{2} V(\xi(t), \eta(t))$ $>V(\xi(t+\theta), \eta(t+\theta))$, then $\dot{V}(\xi(t), \eta(t))<0$ if the matrix

$$
\left(\begin{array}{cc}
\sigma c^{*}-\left(c_{3}(1+\kappa \gamma\|X\|)+\sigma \kappa \gamma\left(\|X\|+\left\|X^{2}\right\|\right)\right) & -\frac{c_{2}+c_{4}(1+\kappa \gamma\|X\|)}{2} \\
-\frac{c_{2}+c_{4}(1+\kappa \gamma\|X\|)}{2} & c_{1}
\end{array}\right)>0 .
$$

Inequality, Eq. (A27) holds if and only if

$$
\begin{gathered}
\sigma c^{*}-\left(c_{3}(1+\kappa \gamma|| X||)+\sigma \kappa \gamma\left(\|X\|+\left\|X^{2}\right\|\right)\right) \\
>\frac{\left(c_{2}+c_{4}(1+\kappa \gamma \| X||)\right)^{2}}{4 c_{1}} .
\end{gathered}
$$

If $\sigma$ is sufficiently large and $\gamma$ sufficiently small, then it is possible to find $\kappa>1$ such that Eq. (A28) holds. Hence, we conclude that there are positive constants $\sigma^{*}$ and $\gamma^{*}$ such that if $\sigma>\sigma^{*}$ and $\sigma \tau<\gamma^{*}$ then the set $\operatorname{ker}\left(I_{k n}-\Pi \otimes I_{n}\right)$ contains a globally asymptotically stable subset.

Proof of Theorem 6. The proof can easily be deducted from the proof of Theorem 5 taking into account that

$$
\begin{aligned}
\left(I_{k m}-\Pi \otimes I_{m}\right) \dot{y}(t)= & \left(I_{k m}-\Pi \otimes I_{m}\right)[a(y(t), z(t)) \\
& \left.-\sigma\left(L \otimes I_{m}\right) y(t-\tau)\right] \\
= & \left(I_{k m}-\Pi \otimes I_{m}\right) a(y(t), z(t)) \\
& -\sigma\left(X \otimes I_{m}\right)\left(I_{k m}-\Pi \otimes I_{m}\right) y(t-\tau) \\
= & \left(I_{k m}-\Pi \otimes I_{m}\right) a(y(t), z(t)) \\
& -\sigma\left(X \otimes I_{m}\right)\left(I_{k m}-\Pi \otimes I_{m}\right) y(t) \\
& +\sigma\left(X \otimes I_{m}\right)\left(I_{k m}-\Pi \otimes I_{m}\right) \int_{-\tau}^{0} \dot{y}(t+s) \mathrm{d} s .
\end{aligned}
$$

Let again

$$
V(\xi(t), \eta(t))=V_{1}(\xi(t))+V_{2}(\eta(t))
$$

with functions $V_{1}$ and $V_{2}$ as in the proof of Theorem 5. Observe that $\dot{V}_{1}$ is the same as in the proof of Theorem 5 and, using the assumptions in the theorem,

$$
\begin{aligned}
& \dot{V}_{2}(\eta) \leq c_{3}|\eta(t)|^{2}+c_{4}|\eta(t)||\xi(t)|-\sigma c^{\prime}|\eta(t)|^{2} \\
& \quad+\sigma \tau \kappa|| X||\left(c_{3}|\eta(t)|^{2}+c_{4}|\eta(t)||\xi(t)|+\sigma \frac{\| X^{2}||}{\| X||}|\eta(t)|^{2}\right),
\end{aligned}
$$

$c_{3}, c_{4}, c^{\prime}>0, \quad$ given that $\kappa^{2} V(\xi(t), \eta(t))>V(\xi(t+\theta)$, $\eta(t+\theta)), \theta \in[-2 \tau, 0]$, with $\kappa>1$. Denote $\gamma=\sigma \tau$ and let $\kappa^{2} V(\xi(t), \eta(t))>V(\xi(t+\theta), \eta(t+\theta)), \theta \in[-2 \tau, 0]$. Then,

$$
\dot{V}(\xi(t), \eta(t))<0
$$

if the matrix

$$
\begin{gathered}
\left(\begin{array}{cc}
\sigma c^{\prime}-\left(c_{3}(1+\kappa \gamma\|X\|)+\sigma \kappa \gamma\left\|X^{2}\right\|\right) & -\frac{c_{2}+c_{4}(1+\kappa \gamma\|X\|)}{2} \\
-\frac{c_{2}+c_{4}(1+\kappa \gamma\|X\|)}{2} & c_{1}
\end{array}\right) \\
>0 .
\end{gathered}
$$

Clearly if $\sigma$ is sufficiently large and $\gamma$ sufficiently small, then it is possible to find $\kappa>1$ such that Eq. (A33) holds. Hence, we conclude that there are positive constants $\sigma^{\prime}$ and $\gamma^{\prime}$ such that if $\sigma>\sigma^{\prime}$ and $\sigma \tau<\gamma^{\prime}$, then the set $\operatorname{ker}\left(I_{k n}-\Pi \otimes I_{n}\right)$ contains a globally asymptotically stable subset.

Proof of Theorem 10. The assumption that $A=$ $\sum_{\ell} \eta_{\ell} \Pi_{\ell}$ is irreducible implies that $A$ is the adjacency matrix of a strongly connected graph. Since the systems are assumed to be strictly semipassive, we can apply Corollary 2 from Ref. 39, which implies that the solutions are ultimately bounded. The claim that $\operatorname{ker}\left(I_{k n}-\Pi_{\ell} \otimes I_{n}\right)$ defines a linear invariant manifold for the coupled systems follows from a slightly modified version of (the proof of) Lemma 3. (Note that all matrices in $\mathbb{P}_{k}$ commute hence all $\Pi_{\ell}$ commute.)

We will prove the theorem by showing that there are constants $\sigma^{\diamond}$ and $\gamma^{\diamond}$ such that if $\sigma>\sigma^{\diamond}$ and $\sigma \tau_{1}<\gamma^{\diamond}$, the set $\operatorname{ker}\left(I_{k n}-\Pi_{1} \otimes I_{n}\right)$ contains a globally asymptotically subset. We will construct a Lyapunov-Razumikhin function of the form Eq. (A2) with

$$
V_{1}(z(t))=z^{\top}(t)\left(I_{k p}-\Pi_{1} \otimes I_{p}\right)^{\top}\left(I_{k} \otimes P\right)\left(I_{k p}-\Pi_{1} \otimes I_{p}\right) z(t)
$$

and

$$
V_{2}(y(t))=y^{\top}(t)\left(I_{k m}-\Pi_{1} \otimes I_{m}\right)^{\top}\left(I_{k m}-\Pi_{1} \otimes I_{m}\right) y(t) .
$$

Following the first part of the proof of Theorem 5, we conclude that there are positive constants $c_{1}, c_{2}$ such that 


$$
\begin{aligned}
& \dot{V}_{1}(z(t)) \leq-c_{1}\left|\left(I_{k p}-\Pi_{1} \otimes I_{p}\right) z(t)\right|^{2} \\
& \quad+c_{2}\left|\left(I_{k p}-\Pi_{1} \otimes I_{p}\right) z(t)\right|\left|\left(I_{k m}-\Pi_{1} \otimes I_{m}\right) y(t)\right| .
\end{aligned}
$$

Note that

$$
\begin{aligned}
\left(I_{k m}-\right. & \left.\Pi_{1} \otimes I_{m}\right) \dot{y}(t) \\
= & \left(I_{k m}-\Pi_{1} \otimes I_{m}\right)[a(y(t), z(t))-\sigma y(t)] \\
& +\sigma \eta_{1}\left(I_{k m}-\Pi_{1} \otimes I_{m}\right) \Pi_{1} y\left(t-\tau_{1}\right) \\
& +\sigma \sum_{\ell\{1\}} \eta_{\ell}\left(I_{k m}-\Pi_{1} \otimes I_{m}\right) \Pi_{\ell} y\left(t-\tau_{\ell}\right) \\
= & \left(I_{k m}-\Pi_{1} \otimes I_{m}\right)[a(y(t), z(t))-\sigma y(t)] \\
& -\sigma \eta_{1}\left(I_{k m}-\Pi_{1} \otimes I_{m}\right) y\left(t-\tau_{1}\right) \\
& +\sigma \sum_{\ell\{1\}} \eta_{\ell} \Pi_{\ell}\left(I_{k m}-\Pi_{1} \otimes I_{m}\right) y\left(t-\tau_{\ell}\right) .
\end{aligned}
$$

In the latter step, we have used the assumption that all matrices in $\mathbb{P}_{k}$ commute and are symmetric. (Recall that for a symmetric permutation matrix, $\Pi^{2}=I$.) Use Leibniz' rule to write

$$
y\left(t-\tau_{1}\right)=y(t)-\int_{t-\tau_{1}}^{t} \dot{y}(s) \mathrm{d} s
$$

and choose $w(s)=\kappa^{2} s, w(s)$ as presented in Eq. (A4), with some constant $\kappa>1$. Denote $\bar{\tau}=\max _{\ell}\left\{\tau_{\ell}\right\}$. Then, using similar arguments as used in the proof of Theorem 5, we conclude that there are constants $c_{3}, c_{4}$ such that

$$
\begin{aligned}
& \dot{V}_{2}(y(t)) \leq\left(c_{3}-\sigma\left(1+\eta_{1}-\kappa\left(1-\eta_{1}\right)\right)\right. \\
& \left.\quad+\gamma_{1} \eta_{1} \kappa\left(c_{3}+2 \sigma\right)\right)\left|\left(I_{k m}-\Pi_{1} \otimes I_{m}\right) y(t)\right|^{2} \\
& \quad+c_{4}\left(1+\kappa \eta_{1} \gamma_{1}\right)\left|\left(I_{k p}-\Pi_{1} \otimes I_{p}\right) z(t)\right|\left|\left(I_{k m}-\Pi_{1} \otimes I_{m}\right) y(t)\right|,
\end{aligned}
$$

with $\gamma_{1}=\sigma \tau_{1}, \quad$ whenever $\quad \kappa^{2} V_{2}(y(t))>V_{2}(y(t+\theta)$, $\theta \in\left[-2 \max \left\{\tau_{1}, \bar{\tau}\right\}, 0\right]$. Since

$$
\frac{1+\eta_{1}}{1-\eta_{1}}>1
$$

because $\eta_{1} \in(0,1)$, we know that there are constants $\kappa>$ $1, \sigma_{1}^{\diamond}>0$ and $\gamma_{1}^{\diamond}>0$, such that if $\sigma>\sigma_{1}^{\diamond}$ and $\gamma<\gamma_{1}^{\diamond}$, then $\dot{V}<0 \quad$ whenever $\quad \kappa^{2} V(z(t), y(t))>V(z(t+\theta), y(t+\theta))$, $\theta \in\left[-2 \max \left\{\tau_{1}, \bar{\tau}\right\}, 0\right]$.

${ }^{1}$ H. M. Smith, "Synchronous flashing of fireflies," Science 82, 151-152 (1935). ${ }^{2}$ J. Buck and E. Buck, "Synchronous fireflies," Sci. Am. 234, 74-85 (1976).

${ }^{3}$ S. H. Strogatz and I. Stewart, "Coupled oscillators and biological synchronization," Sci. Am. 269, 102-109 (1993).

${ }^{4}$ C. S. Peskin, Mathematical Aspects of Heart Physiology (Courant Institute of Mathematical Sciences, New York University, 1975), pp. 268-278.

${ }^{5} \mathrm{C}$. M. Gray, "Synchronous oscillations in neuronal systems: Mechanisms and functions," J. Comput. Neurosci. 1, 11-38 (1994).

${ }^{6}$ C. A. Czeisler, E. D. Weitzman, M. C. Moore-Ede, J. C. Zimmerman, and R. S. Knauer, "Human sleep: Its duration and organization depend on its circadian phase," Science 210, 1264-1267 (1980).

${ }^{7}$ A. T. Winfree, The geometry of biological time, Interdisciplinary Applied Mathematics, 2nd ed. (Springer, 2001).

${ }^{8}$ G. J. L. Naus, R. P. A. Vugts, J. Ploeg, M. J. G. van de Molengraft, and M. Steinbuch, "String-stable CACC design and experimental validation, a frequency-domain approach,” IEEE Trans. Veh. Technol. 59, 4268-4279 (2010).

${ }^{9}$ K. Y. Pettersen, J. T. Gravdahl, and H. Nijmeijer, Group Coordination and Cooperative Control, Group Coordination and Cooperative Control (Springer-Verlag, Berlin, 2006), Vol. 336.

${ }^{10} \mathrm{H}$. Nijmeijer and A. Rodriguez-Angeles, Synchronization of mechanical systems (World Scientific, 2003).

${ }^{11}$ L. M. Pecora and T. L. Carroll, "Synchronization in chaotic systems," Phys. Rev. Lett. 64, 821-824 (1990).

${ }^{12}$ K. M. Cuomo, A. V. Oppenheim, and S. H. Strogatz, "Synchronization of Lorenz based chaotic circuits with applications to communications," IEEE Trans. Circuit Syst., II 40, 626-633 (1993).

${ }^{13}$ H. J. C. Huijberts, H. Nijmeijer, and R. M. A. Willems, "A control perspective on communication using chaotic systems," in Proc. 37th IEEE Conf. Decision and Control (1998), Vol. 2, pp. 1957-1962.

${ }^{14}$ J. Hale, "Diffusive coupling, dissipation, and synchronization," J. Dyn. Differ. Equ. 9, 1-52 (1997).

${ }^{15}$ A. Pogromsky and H. Nijmeijer, "Cooperative oscillatory behavior of mutually coupled dynamical systems," IEEE Trans. Circuit Syst., I 48, $152-162$ (2001).

${ }^{16}$ A. Sherman, J. Rinzel, and J. Keizer, "Emergence of organized bursting in clusters of pancreatic beta-cells by channel sharing," Biophys. J. 54, 411-425 (1988).

${ }^{17}$ G. de Vries, A. Sherman, and H.-R. Zhu, "Diffusively coupled bursters: Effects of cell heterogeneity,” Bull. Math. Biol. 60, 1167-1199 (1998).

${ }^{18}$ C. C. Chow and N. Kopell, "Dynamics of spiking neurons with electrical coupling," Neural Comput. 12, 1643-1678 (2000).

${ }^{19}$ S. Coombes, "Neuronal networks with gap junctions: A study of piecewise linear planar neuron models," SIAM J. Appl. Dyn. Syst. 7, 1101-1129 (2008).

${ }^{20}$ N. Kopell and G. B. Ermentrout, "Mechanisms of phase-locking and frequency control in pairs of coupled neural oscillators," in Handbook of Dynamical Systems, Towards Applications Vol. 2, edited by B. Fiedler, G. Iooss, and N. Kopell (Elsevier, 2002).

${ }^{21}$ T. J. Lewis and J. Rinzel, "Dynamics of spiking neurons connected by both inhibitory and electrical coupling," J. Comput. Neurosci. 14, 283-309 (2003).

${ }^{22}$ J. L. P. Velazquez, "Mathematics and the gap junctions: In-phase synchronization of identical neurons," Int. J. Neurosci. 113, 1095-1101 (2003).

${ }^{23}$ C. W. Wu and L. O. Chua, "Synchronization in an array of linearly coupled dynamical systems," IEEE Trans. Circuit Syst., I 42, 430-447 (1995).

${ }^{24}$ R. v.d. Steen and H. Nijmeijer, "Partial synchronization of diffusively coupled chua systems: An experimental case study," in 1st IFAC Conference on Analysis and Control of Chaotic Systems, Reims, France (2006).

${ }^{25} \mathrm{~A}$. Rodriguez-Angeles and $\mathrm{H}$. Nijmeijer, "Coordination of two robot manipulators based on position measurements only," Int. J. Control 74, 1311-1323 (2001).

${ }^{26}$ D. J. Rijlaarsdam, A. Y. Pogromsky, and H. Nijmeijer, "Synchronization between coupled oscillators: An experimental approach," in Dynamics and Control of Hybrid Mecahnical Systems, World Scientific series on Nonlinear Science, Series B, Vol. 14, edited by G. Leonov, H. Nijmeijer, A. Pogromsky, and A. Fradkov (World Scientific, 2010), pp. 153-165.

${ }^{27}$ S.-J. Chung and J.-J. E. Slotine, "Coorperative robot control and concurrent synchronization of Lagrangian systems," IEEE Trans. Robot. 25, 686-700 (2009).

${ }^{28}$ M. Zanin, J. M. Buldú, and S. Boccaletti, "Networks of springs: A practical approach,” Int. J. Bifurcation Chaos 20, 937-942 (2010).

${ }^{29}$ R. Sipahi, S. Niculescu, C. T. Abdallah, W. Michiels, and K. Gu, "Stability and stabilization of systems with time delay," IEEE Control Syst. 31, 3865 (2011).

${ }^{30}$ B. Liu, D. J. Hill, and J. Yao, "Global uniform synchronization with estimated error under transmission channel noise," IEEE Trans. Circuit Syst., I. 56, 2689-2702 (2009).

${ }^{31}$ M. Zhong and Q.-L. Han, "Fault tolerant master-slave synchronization for Lur'e systems using time-delay feedback control," IEEE Trans. Circuit Syst., I. 56, 1391-1404 (2009).

${ }^{32}$ T. Oguchi and H. Nijmeijer, "A synchronization condition for coupled nonlinear systems with time-delay: A frequency domain approach,” Int. J. Bifurcation Chaos 21, 2525-2538 (2011).

${ }^{33} \mathrm{H}$. Gao, J. Lam, and G. Chen, "New criteria for synchronization stability of general complex dynamical networks with coupling delays," Phys. Lett. A 360, 263-273 (2006). 
${ }^{34} \mathrm{~J}$. Wu and L. Jiao, "Synchronization in complex delayed dynamical networks with nonsymmetric coupling," Physica A 386, 513-530 (2007).

${ }^{35}$ T. Oguchi, H. Nijmeijer, and T. Yamamoto, "Synchronization in networks of chaotic systems with time-delay coupling," Chaos 18, 037108-1037108-14 (2008).

${ }^{36} \mathrm{M}$. Chen, "Synchronization in complex dynamical networks with random sensor delay," IEEE Trans. Circuit Syst., II 57, 46-50 (2010).

${ }^{37}$ N. Chopra and M. W. Spong, Output Synchronization of Nonlinear Systems with Relative Degree One, Lecture Notes in Control and Information Sciences, edited by V. D. Blondel, S. P. Boyd, and H. Kimura (SpringerVerlag, 2008), pp. 51-64.

${ }^{38}$ R. Olfati-Saber and R. M. Murray, "Consensus problems in networks of agents with switching topology and time-delays," IEEE Trans. Autom. Control 49, 1520-1533 (2004).

${ }^{39}$ E. Steur and H. Nijmeijer, "Synchronization in networks of diffusively time-delay coupled (semi-)passive systems," IEEE Trans. Circuit Syst., I 58, 1358-1371 (2011).

${ }^{40}$ W. Kinzel, A. Englert, G. Reents, M. Zigzag, and I. Kanter, "Synchronization in networks of chaotic units with time-delayed couplings," Phys. Rev. E 79, 056207 (2009).

${ }^{41}$ V. Flunkert, S. Yanchuk, T. Dahms, and E. Schöll, "Synchronizing distant nodes: A universal classification of networks," Phys. Rev. Lett. 105, 254101 (2010).

${ }^{42}$ C.-U. Choe, T. Dahms, P. Hövel, and E. Schöll, "Controlling synchrony by delay coupling in networks: From in-phase to splay and cluster states," Phys. Rev. E 81, 025205 (2010).

${ }^{43}$ S. Yanchuk, Y. Maistrenko, and E. Mosekilde, "Partial synchronization and clustering in a system of diffusively coupled chaotic oscillators," Math. Comput. Simul. 54, 491-508 (2001).

${ }^{44}$ A. Pogromsky, G. Santoboni, and H. Nijmeijer, "Partial synchronization: From symmetry towards stability," Physica D 172, 65-87 (2002).

${ }^{45}$ A. Y. Pogromsky, "A partial synchronization theorem," Chaos 18, 037107 (2008) [Erratum: Chaos 19, 049901 (2009)].

${ }^{46}$ V. N. Belykh, I. V. Belykh, and M. Hasler, "Hierarchy and stability of partially synchronous oscillations of diffusively coupled dynamical systems," Phys. Rev. E 62, 6332-6345 (2000).

${ }^{47}$ B. Dionne, M. Golubitsky, and I. Stewart, "Coupled cells with internal symmetry: I. Wreath products,” Nonlinearity 9, 559-574 (1996).

${ }^{48}$ B. Dionne, M. Golubitsky, and I. Stewart, "Coupled cells with internal symmetry: II. Direct products,” Nonlinearity 9, 575-599 (1996).
${ }^{49}$ E. N. Lorenz, "Deterministic nonperiodic flow," J. Atmos. Sci. 20, 130-141 (1963).

${ }^{50}$ It can easily be shown that if the systems have an internal symmetry such that $J f(x)=f(J x)$, and matrices $J$ and $C$ commute, then, under the conditions of lemmas 3 and 4 , the set $\operatorname{ker}(I-\Pi \otimes J)$ defines a linear invariant manifold for the coupled systems.

${ }^{51}$ T. Dahms, J. Lehnert, and E. Schöll, "Cluster and group synchronization in delay-coupled networks,” Phys. Rev. E. 86, 016202 (2012).

${ }^{52}$ L. M. Pecora and T. L. Carroll, "Master stability functions for synchronized coupled systems," Phys. Rev. Lett. 80, 2109-2112 (1998).

${ }^{53}$ A. Y. Pogromsky, "Passivity based design of synchronizing systems," Int. J. Bifurcation Chaos 8, 295-319 (1998).

${ }^{54}$ J. C. Willems, "Dissipative dynamical systems Part I: General theory," Arch. Rational Mech. Anal. 45, 321-351 (1972).

${ }^{55}$ A. V. Pavlov, N. v. d. Wouw, and H. Nijmeijer, Uniform Output Regulation of Nonlinear Systems (Birkhäuser, Berlin, 2006).

${ }^{56}$ B. P. Demidovich, Lectures on Stability Theory (Nauka-Moscow, 1967) (in Russian).

${ }^{57}$ T. A. Burton, Stability and Periodic Solutions of Ordinary and Functional Differential Equations, Mathematics in Science and Engineering Vol. 178 (Academic Press, Orlando, Florida, 1985).

${ }^{58}$ C. I. Byrnes, A. Isidori, and J. C.Willems, "Passivity, feedback equivalence, and the global stabilization of minimum phase nonlinear systems," IEEE Trans. Autom. Control 36, 1228-1240 (1991).

${ }^{59}$ J. L. Hindmarsh and R. M. Rose, "A model for neuronal bursting using three coupled differential equations," Proc. R. Soc. London, Ser. B 221, 87-102 (1984).

${ }^{60}$ E. Steur, I. Tyukin, and H. Nijmeijer, "Semi-passivity and synchronization of diffusively coupled neuronal oscillators," Physica D 238, 2119-2128 (2009).

${ }^{61}$ R. A. Horn and C. R. Johnson, Matrix Analysis, 6th ed. (Cambridge University Press, 1999).

${ }^{62}$ S. Boccaletti, V. Latora, Y. Moreno, M. Chavez, and D.-U. Hwang, "Complex networks: Structure and dynamics," Phys. Rep. 424, 175-308 (2006).

${ }^{63}$ T. Ishizaki, K. Kashima, J.-I. Imura, and K. Aihara, "Reaction-diffusion clustering of single-input dynamical networks," in Proc. 50th IEEE Conf. Decision and Control (2011), pp. 7837-7842.

${ }^{64}$ J. K. Hale and S. M. Verduyn Lunel, Introduction to Functional Differential Equations, Applied Mathematical Sciences Vol. 99 (Springer-Verlag, 1993). 